\title{
TITLE:
}

\section{On the Resolution of Hypersurface Singularities}

$\operatorname{AUTHOR}(S):$

OKA, Mutsuo

CITATION:

OKA, Mutsuo. On the Resolution of Hypersurface Singularities. 数理解析 研究所講究録 1984, 535: 210-256

ISSUE DATE:

1984-09

URL:

http://hdl.handle.net/2433/98656

RIGHT: 
On the Resolution of Hypersurface Singularities

By Mutsuo OKA 岡 睦雄

東亲工業规学理学部。数学教室。

§1. Introduction.

Let $f\left(z_{1}, \ldots, z_{n+1}\right)$ be a germ of an analytic function at the origin such that $f(0)=0$ and $f$ has an isolated critical point at the origin. We assume that $f$ has a non-degenerate Newton boundary. Let $V$ be a germ of hypersurface $f^{-1}(0)$ at the origin. We will study the resolution of $v$. Let $\Gamma^{*}(f)$ be the dual Newton diagram and let $\Sigma^{*}$ be a simplicial subdivision. It is well-known that there is a canonical resolution $\pi: \tilde{v} \rightarrow V$ which is associated with $\Sigma^{*}$. ([5]). However the process to get $\Sigma^{*}$ from $\Gamma^{*}(f)$ is not unique and a "bad" $\quad \Sigma^{*}$ produces unnecessary exceptional divisors.

The purpose of this paper is to study the topology of the exceptional divisors using a "canonical" subdivision $\Sigma^{*}$. For example, if $n=2$, there is a "unique" subdivision $\Sigma^{*}$ so that the resolution graph is obtained by a canonical surgery from $S_{2} \Gamma^{*}(f) \quad\left(=2\right.$ skeleton of $\Gamma^{*}(f)$ which we consider as a graph by a plane section). Namely we have:

(I). For a vertex $P$ of $S_{2} \Gamma^{*}(f)$ which corresponds to a compact two dimensional face $\Delta(P)$ of the Newton boundary $\Gamma(f)$, there is an exceptional divisor $E(P)$ which is a Riemann surface of genus $g(\dot{P})$. Here $g(P)$ is equal to the number of integral points of $\Delta(P)-\partial \Delta(P)$.

(II). A line segement $\overline{P Q}$ of $S_{2} \Gamma^{*}(f)$ is replaced by $r(P, Q)+1$ copies of a chain of rational curves

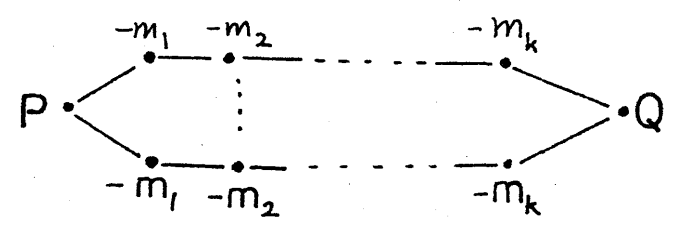


where $r(P, Q)$ is the number of integral points on $\Xi-\partial \equiv$,

$\Xi=\Delta(P) \cap \Delta(Q)$. The continuous fraction $m_{1}-\frac{1}{m_{2}-}$. $\because-\frac{1}{m_{k}}$

is canonically associated to $P$ and $Q$. (See Theorem (6.1).)

This paper consists of the following sections.

\$2. Newton boundary and the dual Newton diagram.

§3. Canonical simplicial subdivision.

$\$ 4$. The resolution of $V$.

\$5. Topology of the exceptional divisors.

\$6. The surface singularity.

§7. Three dimensional singularity.

\$2. Newton boundary and the dual Newton diagram.

Let $f\left(z_{1}, \ldots, z_{n+1}\right)=\Sigma a_{v} z^{\nu}$ be the Taylor expansion of $f$ where $z^{\nu}=z_{1}{ }^{\nu} \ldots z_{n+1} v_{n+1}$ as usual. Recall that the Newton boundary $\Gamma(f)$ is the union of compact faces of $\Gamma_{+}(f)$ where $\Gamma_{+}(f)$ is the convex hull in $R^{n+1}$ of the union of the subsets $v+\left(\mathbb{R}^{+}\right)^{n+1}$ for $v$ such that $a_{v} \neq 0$.

$\Gamma(f)$ has a natural polyhedral decomposition. For any closed face $\Delta$ (of any dimension) of $\Gamma(f)$, we associate the polynomial $f_{\Delta}(z)=\sum_{v \in \Delta} a_{v} z^{\nu}$. We say that $f$ is non-degenerate on $\Delta$ if $\frac{\partial f_{\Delta}}{\partial z_{1}}=\ldots=\frac{\partial f_{\Delta}}{\partial z_{n+1}}=0$ has no solution in $\left(\mathbb{C}^{*}\right)^{n+1}$. We say that $f$ is non-degenerate if $f$ is non-degenerate on any face $\Delta$ of $\Gamma(f) . \quad([6],[14])$. 
Let $\mathrm{N}^{+}$be the space of positive vectors of the dual space of $R^{\mathrm{n}+l}$. For any vector $a=\left(\begin{array}{c}a_{1} \\ \vdots \\ a_{n+1}\end{array}\right)$ of $N^{+}$, we associate the linear function $a(x)=$ $\sum a_{i} x_{i}$ on $\Gamma_{+}(f)$. Let $d(a)$ be the minimum value of a on $\Gamma_{+}(f)$ and let $\Delta(a)=\left\{x \in \Gamma_{+}(f) ; a(x)=d(a)\right\}$. We introduce an equivalent relation $\sim$ on $\mathrm{N}^{+}$by $a \sim b$ if and only $\Delta(a)=\Delta(b)$. For any face $\Delta$ of dimension $k$ of $\Gamma_{+}(f)$, there is an equivalence class $\Delta^{*}$ of dimension $(n+1-k)$ which is defined by $\Delta^{*}=\left\{a \varepsilon N^{+} ; \Delta(a)=\Delta\right\}$. The collection of $\Delta^{*}$ gives a polyhedral decomposition $\Gamma^{*}(f)$ of $N^{+}$mpich we call the dual Newton diagram of $f$. We identify $\Gamma^{*}(f)$ and its projection on the hyperplane $L=\left\{x_{1}+\ldots+x_{n+1}=1\right\}$. Thus a vertex $P$ of $\Gamma^{*}(f)$ is a primitive integral vector such that $\Delta(P)$ is of dimension $n$. If $P$ is strictly positive, i.e. each coordinate of $P$ is positive, $\Delta(P)$ is a compact face of $\Gamma(f)$.

Example (2.1). Let $f(x, y, z)=x^{4}+y^{4}+z^{4}+x y z$. Then $\Gamma(f)$ has three faces of maximal dimension and $\Gamma^{*}(f)$ is the following.

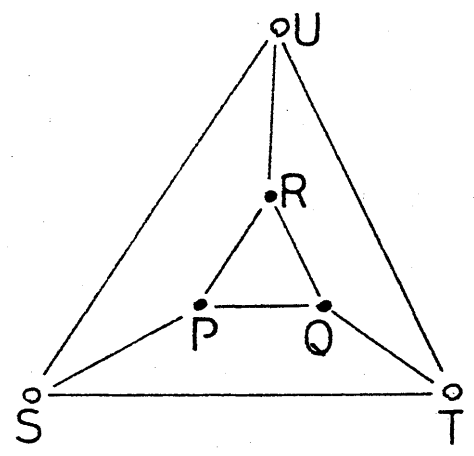

$$
\begin{aligned}
& P=t^{t}(2,1,1), S=(1,0,0) \\
& Q={ }^{t}(1,2,1), T=t(0,1,0) \\
& R={ }^{t}(1,1,2), U=^{t}(0,0,1)
\end{aligned}
$$

Figure (2.1)

For any $P \varepsilon N^{+}$, we define the support of $P$ by $\Delta(P)^{*}$.

We say a polyhedral subdivision $\Sigma^{*}$ of $\Gamma^{*}(f)$ is a simplicial subdivision if the following conditions are satisfied. ([ 5 ]). 
(i) $\Sigma^{*}$ is a subdivision by the cones over the simplexes $\sigma=\left(P_{1}, \ldots, P_{k+1}\right)$ where $P_{1}, \ldots, P_{k+1}$ are primitive vectors and they can be extended to a basis of $\mathbb{Z}^{n+l}$. We identify the cone over a simplex $\sigma$ and $\sigma$ itself. In particular, any $n$-simplex $\sigma=\left(P_{1}, \ldots, P_{n+1}\right)$ with $\left.P_{i}=t_{\left(p_{1, i}\right.}, \ldots, p_{n+1, i}\right)$ satisfies $\operatorname{det}\left(p_{i, j}\right)= \pm l$. The intersection of two simplexes is a simplex. (ii) For any $n$-simplex as above, there exists a permutation $\tau$ of $\{1, \ldots, n+1\}$ such that

$$
\Delta\left(P_{\tau(1)}\right) \supset \Delta\left(P_{\tau(2)}\right) \supset \ldots \supset \Delta\left(P_{\tau(n+1)}\right) \text {. }
$$

Remark (2.3). The condition (2.2) can be replaced by a weaker condition but (2.2) makes the intersection of exceptional divisors easier to be understood. (See Lemma (4.7).)

§3. Canonical simplicial subdivision.

Let $p_{i}={ }^{t}\left(p_{1, i}, \ldots, p_{n+1, i}\right) \quad(i=1, \ldots, k)$ be given integral vectors of $\mathrm{N}^{+}$. We define a non-negative integer $\operatorname{det}\left(P_{1}, \ldots, P_{k}\right)$ by the greatest common divisor of all $k \times k$ minors of the matrix $\left(p_{j, i}\right)$ and we call $\operatorname{det}\left(P_{1}, \ldots, P_{k}\right)$ the determinant of $P_{1}, \ldots, P_{k}$.

Lemma (3.1). Let $A=\left(a_{i, j}\right)$ be a unimodular matrix. Then we have $\operatorname{det}\left(P_{1}, \ldots, P_{k}\right)=\operatorname{det}\left(A P_{1}, \ldots, A P_{k}\right)$.

The proof is an easy exercise of linear algebra.

Lemma (3.2). Let $P_{1}, \ldots, P_{k}$ ) be given integral vectors such that $\operatorname{det}\left(P_{1}, \ldots, P_{k}\right)= \pm 1$. Then there exist integral vcetors $P_{k+1}, \ldots, P_{n+1}$ such that $\operatorname{det}\left(P_{1}, \ldots, P_{n+1}\right)= \pm 1$.

Proof. Let $M$ be the subgroup of $\mathbb{2}^{n+1}$ generated by $P_{1}, \ldots, P_{k}$. Then by the structure theorem of a finitely generated abelian group, there is a 
subgroup $M^{\prime}$ of rank $k$ such that $M C M^{\prime}$ and ' $M$ ' is a direct summand of $\mathbb{Z}^{n+1}$. Then the assumption that $\operatorname{det}\left(P_{1}, \ldots, P_{k}\right)=1$ clearly implies $M^{\prime}=M^{\prime}$.

(I). Division of a line segment.

Let $P=\left(\begin{array}{l}p_{1} \\ \vdots \\ p_{n+1}\end{array}\right)$ and $Q=\left(\begin{array}{l}a_{1} \\ \vdots \\ q_{n+1}\end{array}\right)$ be given distinct primitive integral

Lemma (3.3). Let $c=\operatorname{det}(P, Q)$.

(i) Any integral vector $P_{1}$ on the segment $\overline{P Q}$ such that $\operatorname{det}\left(P, P_{1}\right)=1$ can be written as

$$
P_{1}=\frac{1}{C} Q+\frac{C_{1}}{C} P
$$

for some integer $c_{1} \geqq 0$.

(ii) There exists a unique $c_{1}$ such that $0 \leqq c_{1}<c$.

(iii) $P_{1}$ is necessarily primitive.
Proof. By Lemmas (3.1) and (3.2), we may assume that $Q=\left(\begin{array}{c}1 \\ 0 \\ \vdots \\ 0\end{array}\right)$.

Then $c$ is nothing but g.c.d( $\left.p_{2}, \cdots, p_{n+1}\right)$. Let $P_{1}=\lambda P+\mu Q(\lambda \geqq 0, \mu \geqq 0)$ and assume that $P_{1}$ is an integral and $P_{1}$ satisfies $\operatorname{det}\left(P, P_{1}\right)=1$. As $\operatorname{det}\left(P, P_{1}\right)=\mu \cdot \operatorname{det}(P, Q)=\mu c=1$, we have $\mu=\frac{1}{C} \cdot$ As $P_{1_{C}}$ is integral, $\lambda \mathrm{p}_{i} \varepsilon \mathbb{Z}$ for $i=2, \ldots, n+1$ and this implies that $\lambda=\frac{{ }^{c_{1}}}{c}$ for some $c_{1} \geqq 0$ and $c_{1} p_{1}+1=0$ modulo $c$. The last equation has a unique solution in $0 \leqq c_{1}<c$ as g.c.d. $\left(p_{1}, c\right)=$ g.c.d. $\left(p_{1}, \ldots, p_{n+1}\right)=1$. The assertion (iii) is immediate from the assumption $\operatorname{det}\left(P, P_{1}\right)=1$. Note that $c_{1}=0$ if and only if $c=1$.

Remark (3.4). By the abuse of language, we say that $P_{1}=\lambda P+\mu Q$ is on the line segment $\overline{P Q}$ if the normalized vector $P_{i}=\frac{\lambda}{\lambda+\mu} P+\frac{\mu}{\lambda+\mu} Q$ is on $\overline{P Q}$. This is the case if and only if $\lambda \geqq 0, \mu \geqq 0$.

Definition (3.5). Let $\overline{P Q}$ be a line segment of $S_{2} \Gamma^{*}(f)$. Here $S_{k} \Gamma^{*}(f)$ is the k-skeleton of $\Gamma^{*}(f)$. We say that 
the primitive vectors $P_{1}, \ldots, P_{k}$ on $\overline{P Q}$ are the canonical primitive sequences if the following is satisfied.

(i) If $c=\operatorname{det}(P, Q)=1, k=1$ and $P_{1}=P+Q$.

(ii) If $c>1$, there are non-negative integers $c=c_{0}>c_{1}>\ldots>c_{k}=1, c_{k+1}=0$ such that

$$
P_{i+1}=\frac{1}{c_{i}} Q+\frac{c_{i+1}}{c_{i}} P_{i} \quad(i=0, \ldots, k)
$$

where $P_{0}=P$ and $P_{k+1}=Q$.

The condition ( $i$ ) is to satisfy (2.2).

Lemma (3.6). Assume that $c=\operatorname{det}(P, Q)>1$ and let $P_{1}, \ldots, P_{k}$ be the canonical primitive sequence of the line segment $\overline{P Q}$. Let $c_{i}(i=0, \ldots, k+1)$ be as in (3.5) and let $m_{i}=\frac{c_{i-1}+c_{i+1}}{c_{i}}(i=1, \ldots, k)$. Then $m_{i}$ is an integer such that $m_{i} \geqq 2$ and the continuous fraction

$$
m_{1}-\frac{1}{m_{2}-}
$$

is equal to $\frac{c}{c_{1}}$.

Let $P_{i}=\left[\begin{array}{c}p_{1, i} \\ \vdots \\ p_{n+1, i}\end{array}\right]$. Then $m_{i}$ is equal to $\frac{p_{j, i-1}+p_{j, i+1}}{p_{j, i}}$ for any $j=1, \ldots, n+1$.

Proof. We prove the assertion by the induction on $k$. Assume that $k=1$. Then $P_{1}=\frac{1}{c} Q+\frac{1}{c} P$. Thus $m_{1}=\frac{c+0}{c_{1}}=c$. As $c p_{j, 1}=q_{j}+p_{j}$, we have $c=\frac{p_{j}+q_{i}}{p_{j_{1}}}$. Assume that $k>1$. Then as $P_{1}=\frac{Q}{c}+\frac{c_{1}}{c} P$ and $P_{2}=\frac{Q}{c_{1}}+\frac{c_{2}}{c_{1}} P_{1}$, we have that $\operatorname{det}\left(P, P_{2}\right)=\frac{1}{c_{1}} \operatorname{det}\left(P, Q+C_{2} P_{1}\right)=\frac{1}{c_{1}}\left(1+\frac{C_{2}}{C}\right) \operatorname{det}(P, Q)=\frac{C+C_{2}}{C_{1}}=m_{1}$. 
Thus $m_{1}$ is an integer and $m_{1} \geq 2$. We can write $P_{1}=P / m_{1}+P_{2} / m_{1}$. This implies that $P_{1}$ is the canonical primitive sequence for $\overline{\mathrm{PP}}_{2}$. Now we have $m_{1}-\frac{1}{m_{2}-}=\frac{c+c_{2}}{c_{1}}-\frac{1}{c_{1} / c_{2}}=c / c_{1}$, completing the proof of the $-\frac{1}{m_{k}}$

first assertion. The second assertion is obvious from the equality:

$\left(c_{i-1}+c_{i+1}\right) P_{i}=c_{i}\left(P_{i-1}+P_{i+1}\right)$.

Remark (3.7). Lemma (3.6) is true for every primitive sequence $P_{1}, \ldots$, $P_{S}$ on $\overline{P Q}$ such that $\operatorname{det}\left(P_{i-1}, P_{i}\right)=1$ for $i=1, \ldots, s+1 \quad\left(P_{0}=P\right.$ and $P_{S+1}$

=Q). exept that $m_{i} \geqq 1$ in this case. For example, $\left[m_{1}+1,1, m_{2}+1, m_{3}, \ldots, m_{k}\right]$

$=\left[m_{1}, \ldots, m_{k}\right]$. Here we write $r_{1}-\frac{1}{r_{2}-\cdot-\frac{1}{r_{t}}}=\left[r_{1}, \ldots, r_{t}\right]$ for brevity's

sake. Note that if $c=\operatorname{det}(P, Q)>1$, a primitive sequence $P_{1}, \ldots, P_{S}$ is canonical if and only if $m_{i} \geqq 2$ for each $i$, by the second expression of $m_{i}$. In particular, if $P_{l}, \ldots, P_{k}$ is the canonical primitive sequence of $\overline{P Q}$, $P_{k}, \ldots, P_{1}$ is the canonical primitive sequence of $\overline{Q P}$. The ambiguity of the continuous fraction expression $\left[r_{1}, \ldots, r_{s}\right]$ for $r_{i} \geq 1$ corresponds to the existence of the exceptional curve of the first kind. (See §6.)

(II) Division of $S_{3} \Gamma^{*}(f)$.

Let $\xi$ be a closed cell of dimension 2 with vertices $P, \ldots$, S. There is no unique way to divide $\xi$ into simplexes with determinant 1 . We first take canonical primitive sequence on the boundaries and divide $\xi$ into triangles without adding any other vertices. The determinant need not be equal to one. 
Lemma (3.8). Let $\triangle$ be a triangle with primitive integral vectors $P, Q$, $R$ as vertices. Let $C=\operatorname{det}(P, Q, R)$ and assume that $C>1$ and $\operatorname{det}(P, Q)=$ $\operatorname{det}(P, R)=1$. Then there are unique $c_{1}$ and $d_{1}$ such that (i) $0<c_{1}<c$ and $0 \leqq d_{1}<c$. (ii) The point defined by $T_{1}=\frac{1}{c} R+$ $\frac{c_{1}}{c} Q+\frac{d_{1}}{c} P$ is an integral point which satisfies $\operatorname{det}\left(P, Q, T_{1}\right) \cdot\left(\right.$ iii) $T_{1}$ divide $\Delta$ into three (two if $d_{1}=0$ ) triangles with $\operatorname{det}\left(P, Q, T_{1}\right)=1, \operatorname{det}\left(P, T_{1}, R\right)$ $=c_{1}$ and $\operatorname{det}\left(Q, T_{1}, R\right)=d_{1}$.

Proof. By Lemma (3.1) and Lemma (3.2), we may assume that $P={ }^{t}(1,0, \ldots, 0)$, $Q={ }^{t}(0,1,0, \ldots, 0)$ and $R={ }^{t}\left(r_{1}, r_{2}, r_{3}, 0, \ldots, 0\right)$. Thus $c=r_{3} \cdot T_{1}$ is an integral point if and only if $c_{1}+r_{2} \equiv 0, d_{1}+r_{1} \equiv 0$ modulo c. Clearly this has a unique solution in $0 \leqq c_{1}<c$ and $0 \leqq d_{1}<c$. If $c_{1}=0$, $T_{1}$ is on $\overline{P R}$ and we have a contradiction $1=\operatorname{det}(P, R)=c \operatorname{det}\left(P, T_{1}\right)$. The other assertions are obvious.

Note that $\operatorname{det}\left(P, T_{1}\right)=1$ by $\operatorname{det}\left(P, Q, T_{1}\right)=1$. Thus we can do the same operation on the triangle $\Delta_{1}=\Delta\left(P, T_{1}, R\right)$ if $c_{1}>1$. By the induction, we get

Lemma (3.9). Assume that $c>1$. There exists unique integers $k, c_{1}, \ldots$, $c_{k}$ and $d_{1}, \ldots, d_{k}$ such that $c=c_{0}>c_{1}>\ldots>c_{k}=1$ and $0 \leqq d_{i}<c_{i-1}$ and $T_{i}=R / c_{i-1}+c_{i} T_{i-1} / c_{i-1}+d_{i} P / c_{i-1}(i=1, \ldots, k)$ are integral points with $\operatorname{det}\left(P, T_{i-1}, T_{i}\right)=1$ where $T_{0}=Q$. (See Figure (3.10).)

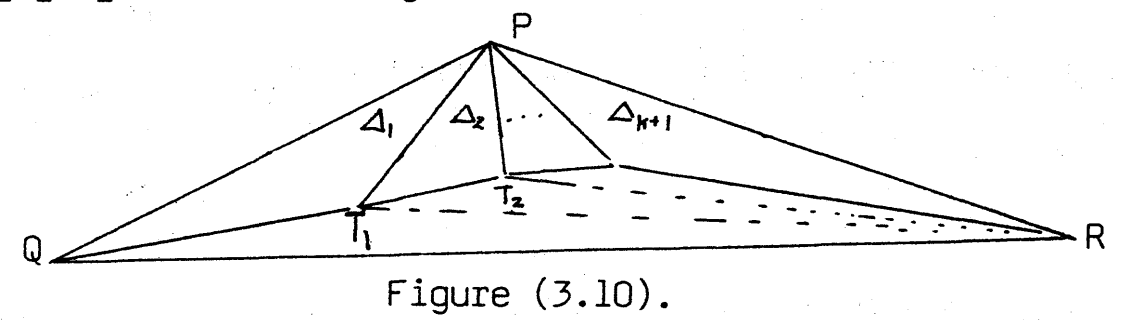

We say that $\Delta_{i}=\left(P, T_{i-1}, T_{i}\right)(i=1, \ldots, k+1)$ are the canonical simplicial subdivision of the triangle $(P, Q, R)$ at $P .\left(T_{k+1}=R\right)$. In the case $c=1$, we take 
one vertex $T=Q+R$ if $\Delta(Q) \downarrow \Delta(R)$ and $\Delta(Q) \ngtr \Delta(R)$. Otherwise we do not need any subdivision.

We return to the subdivision of $\xi$. Take any triangle $\Delta\left(P^{\prime}, Q^{\prime}, R^{\prime}\right)$. If $\operatorname{det}\left(P^{\prime}, Q^{\prime}\right) \neq 1$, we take canonical primitive sequences on $\overline{P^{\prime} Q^{\prime}}$, say $P_{1}, \ldots, P_{k}$, and divide $\Delta\left(P^{\prime}, Q^{\prime}, R^{\prime}\right)$ into $k+1$ triangles with the property $\operatorname{det}\left(P_{i}, P_{i+1}, R^{\prime}\right)$ $<\operatorname{det}\left(P^{\prime}, Q^{\prime}, R^{\prime}\right)$. If $\operatorname{det}\left(P^{\prime}, Q^{\prime}\right)=1$ and $\Delta\left(P^{\prime}\right)$ and $\Delta\left(Q^{\prime}\right)$ are not compatible with (2.2), we take one vertex $P_{1}=P^{\prime}+Q^{\prime}$. Thus we may assume that $\operatorname{det}\left(P^{\prime}, Q^{\prime}\right)$ $=1$ and $\Delta\left(P^{\prime}\right)$ and $\Delta\left(Q^{\prime}\right)$ are compatible with (2.2). Then we do the same opera tion on $\overline{P^{\prime}} R^{\prime}$. As the determinants of the triangles are monotone decreasing under the above operation, after a finite number of operations we come to the situation of Lemma (3.8). Then we apply Lemma(3.8) and so on. Thus by the finite operations we can divide $\xi$ into simplicial triangles i.e. with determinant one and the condition (2.2). By this argument, we can divide $S_{3} \Gamma^{*}(f)$ into simplicial triangles so that the restriction of the subdivision to the $S_{2} \Gamma^{*}(f)$ is the canonical one which is constructed in (3.5).

The subdivision of $\Gamma^{*}(f)$ can be done by the inductive argument. Suppose we have a simplicial sudivision of $S_{m} \Gamma^{*}(f)$. First we divide $S_{m+1} \Gamma^{*}(f)$ into simplexes with determinant not necessarily one without adding any vertex on $\mathrm{S}_{\mathrm{m}} \Gamma^{*}(f)$. Then the following Lemma plays the same role as above.

Lemma (3. 1 ). Let $\underset{i}{\Delta}$ be a simplex with vertices $P_{1}, \ldots, P_{m+1}$ and assume that $\operatorname{det}\left(P_{1}, \ldots, \stackrel{i}{P}_{i}, \ldots, P_{m+1}\right)=1$ and $c=\operatorname{det}\left(P_{1}, \ldots, P_{m+1}\right)>1$ $(i=2, \ldots, m+1)$. Then there exists unique integers $c_{1}, \ldots, c_{m}$ such that the vertex $T$ defined by

$$
T=c_{1} P_{1} / c+\ldots+c_{m} \cdot P_{m} / c+P_{m+1} / c
$$

is an integral point and $0<c_{i}<c$ for $i \neq 1$ and $c>c_{1} \geqq 0$. $T$ divides 
$\Delta$ into $m+1$ simplexes wi.th respective determinant $c_{1}, \ldots, c_{m}$ and 1 .

The proof is completely parallel to that of Lemma (3.8). The details are left for the reader. See also [5] for a further information.

$\$ 4$. The resolution of $V$.

Let $f$ be an analytic function with an isolated critical point at the origin. We assume that $f$ has a non-degenerate Newton boundary and let $\sum *$ be a given simplicial subdivision of $\Gamma^{*}(f)$.

For any $n$-simplex $\sigma=\left(P_{1}, \ldots, P_{n+1}\right)=\left(p_{i j}\right)$, we associate $(n+1)$ - dimensional Euclidean space $\mathbb{C}_{\sigma}^{n+1}$ with coordinate $\left(y_{\sigma, 1}, \ldots, y_{\sigma, n+1}\right)$ and holomorphic mapping $\pi_{\sigma}: \mathbb{C}_{\sigma}^{n+1} \longrightarrow \mathbb{C}^{n+1}$ which is defined by $z_{i}=y_{\sigma, 1}^{p_{i, 1}} y_{\sigma, 2}^{p_{i, 2}}, \ldots, y_{\sigma}^{p_{i, n+1}}$. By the abuse of the notation, we write $z=\left(y_{\sigma}\right)^{\sigma}$. Let $x$ be the union of $c_{\sigma}^{n+1}$ which are glued along the images of $\pi_{\sigma}$. Let $\pi: X \rightarrow \mathbb{C}^{n+1}$ be the projection map and let $\tilde{V}$ be the closure of $\pi^{-1}\left(V_{\cap}\left(\mathbb{C}^{*}\right)^{n+1} \quad\right.$ It is well-known that $\pi: \widetilde{V} \rightarrow V$ is a resolution of $\vee([5]) .(n \geqq 2)$

Let $d_{i}=d\left(P_{i}\right)$ and $\Delta_{i}=\Delta\left(P_{i}\right)$ be as in $\$ 2$. The ordering of $P_{1}, \ldots, P_{n+1}$ is so chosen that

$$
\text { (4.1). } \quad \Delta_{1} \supset \Delta_{2} \supset \ldots>\Delta_{n+1} .
$$

We define $g_{\Delta_{i}}\left(y_{\sigma, 1}, \ldots, y_{\sigma, n+1}\right)=f_{\Delta_{i}}\left(\pi_{\sigma}\left(y_{\sigma}\right)\right) / \underset{j=1}{n+1} y_{j, j}^{d j}$. It is easy to see that $g_{\Delta_{i}}$ is a function of $n+1-i$ variables $y_{\sigma, i+1}, \ldots, y_{\sigma, n+1}$. If $P_{i}$ is strictly positive, $g_{\Delta_{i}}$ is a polynomial. We can write 
$\pi_{\sigma}^{*} f\left(y_{\sigma}\right)=y_{\sigma, 1}^{d_{1}} \ldots y_{\sigma, n+1}^{d_{n+1}} f_{\sigma}\left(y_{\sigma}\right)$. By the definition of $\widetilde{v}, \tilde{v}_{\cap} \mathbb{C}_{\sigma}^{n+1}=$ $\left\{f_{\sigma}=0\right\}$ and $f_{\sigma}\left(y_{\sigma, 1}, \ldots, \stackrel{j}{0}, \ldots y_{\sigma, n+1}\right)=g_{\Delta_{i}}\left(y_{\sigma, j+1}, \ldots, y_{\sigma, n+1}\right)$.

Thus we have

(4.2). $\tilde{V} \cap\left\{y_{\sigma, j}=0\right\} \neq \emptyset$ if and only if $\operatorname{dim} \Delta_{i}>0$.

Recall that $=S_{k} \Gamma^{*}(f)$ is the union of cells of $\Gamma^{*}(f)$ whose dimension is less than $k+1$, and we call $S_{k} \Gamma^{*}(f)$ the $k$ - skeleton of $\Gamma *(f)$.

Remark? The dimension of a cell decreases by 1 if we consider the section with the hyperplane $x_{1}+\ldots+x_{n+1}=1$.

Corollary (4.4). Assume that $\sigma \cap S_{n} \Gamma^{*}(f)=\emptyset$. Then $\widetilde{v} \cap \mathbb{C}_{\sigma}^{n+1} \subset\left(\mathbb{C}_{\sigma}^{*}\right)^{n+1}$. Thus no exceptional divisor is in this chart.

Let $\sigma$ be as above and let $E\left(P_{i} ; \sigma\right)=\widetilde{V}_{\cap}\left\{y_{\sigma, i}=0\right\}=\left\{\left(y_{\sigma, 1}, \ldots, y_{\sigma, n+1}\right)\right.$; $\left.y_{\sigma, i}=0, g_{\Delta_{i}}\left(y_{\sigma, i+1}, \ldots, y_{\sigma, n+1}\right)=0\right\}$. The smoothness of $\widetilde{V}$ and $E\left(P_{i} ; \sigma\right)$ in a neighborhood of $\pi^{-1}(0)$ is easily derived from the non-degeneracy assumption of the Newton boundary. By the definition of $\pi_{\sigma}$, we have (4.5). $\pi_{\sigma}\left(E\left(P_{i} ; \sigma\right)\right)=\{0\}$ if and only if $P_{i}$ is strictly positive. Now we study the glueing map between $\mathbf{c}_{\sigma}^{n+1}$ and $\mathbb{C}_{\tau}^{n+1}$. Let $\sigma$ $=\left(P_{1}, \ldots, P_{n+1}\right)$ and $\tau=\left(Q_{1}, \ldots, Q_{n+1}\right)$. We can write $Q_{i}=\sum_{j=1}^{n+1} \lambda_{j i} P_{j} \quad$ for any $i=1, \ldots, n+1$.

Then $y_{\sigma}=\pi_{\sigma}^{-1} \circ \pi_{\tau}\left(y_{\tau}\right)=\pi_{\sigma}^{-1} \circ \tau\left(y_{\tau}\right)$ and $\sigma^{-1} \tau$ is the matrix $\Lambda=\left(\lambda_{i j}\right)$. Namely we have
$(4.6)$.
$y_{\sigma, i}=y_{\tau, 1}^{\lambda_{i, 1}} \quad \cdots y_{\tau, n+1}^{\lambda_{i, n+1}}$
$(i=1, \ldots, n+1)$.

In particular, if $P_{j}=Q_{i}$ we have 


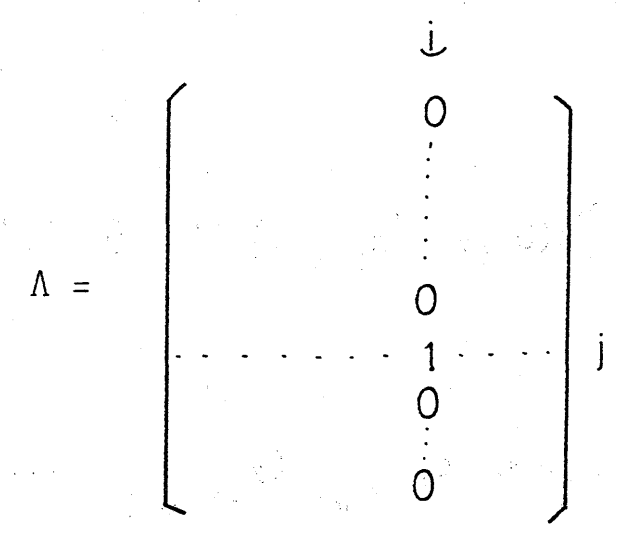

Let $\Lambda^{\prime}=\left(\lambda_{\alpha, \beta}\right)_{\alpha \neq j, \beta \neq i}$. Then $\Lambda^{\prime}$ is a unimodular matrix and $E\left(Q_{i}, \tau\right)$ and $E\left(P_{j} ; \sigma\right)$ are glued by $y_{\tau}^{\prime}=\left(y_{\tau, 1}, \ldots, \stackrel{\dot{i}}{0}, \ldots, y_{\tau, n+1}\right) \rightarrow y_{\sigma}^{\prime}=\left(y_{\tau}^{\prime}\right)^{\prime}$. Thus for each $P \varepsilon \Sigma$ with $\operatorname{dim} \Delta(P)>0$, the union of $E(P ; \sigma)$ for simplexes $\sigma$ such that $P \varepsilon \sigma$ is a divisor of $V$ and we denote it by $E(P) . E(P)$ is a compact exceptional divisor if and only if $P$ is strictly positive. If $P$ is not strictly positive, $\pi(E(P)) \neq\{0\}$. The topology of $E(P)$ will be studied in the following sections.

We say that vertices $P_{1}, \ldots, P_{k}$ in $\Sigma^{*}$ are adjacent if there is an n-simplex $\sigma$ of $\Sigma^{*}$ such that $P_{i} \in \sigma$ for $i=1, \ldots, k$.

The following criterion for the intersection is important.

Lemma (4.7). Let $P_{1}, \ldots, P_{k}$ be mutually distinct vertices of $\Sigma^{*}$ with $\operatorname{dim} \Delta\left(P_{i}\right)>0$ for $i=1, \ldots, k$. Then the intersection $E\left(P_{1}\right) \cap \cdots \cap E\left(P_{k}\right)$ is non-empty if and only if $P_{1}, \ldots, P_{k}$ are adjacent. If $P_{1}, \ldots, P_{k}$ are adjacent and if $\bigcap_{i=1}^{k} \Delta\left(P_{i}\right)$ is compact, $E\left(P_{1}\right) \cap \cdots \cap E\left(P_{k}\right)$ is a compact manifold of dimension $n-k$.

Proof. $E\left(P_{i}\right) \cap \mathbb{C}_{\sigma}^{n+1} \neq \emptyset$ if and only if $\sigma \ni P_{i}$. Thus the first assertion is obvious by $(2.2)$. Let $\sigma=\left(Q_{1}, \ldots, Q_{n+1}\right)$ be a simplex such that

(*). $\Delta\left(Q_{1}\right) \supset \Delta\left(Q_{2}\right) \supset \ldots \supset \Delta\left(Q_{n+1}\right)$

and $P_{i}=Q_{\nu_{i}}$ for $v_{1}<v_{2}<\cdots<v_{k}$.

In $\mathbb{C}_{\sigma}^{n+1}, E\left(P_{i} ; \sigma\right)$ is defined by 


$$
\left.E\left(P_{i} ; \sigma\right)=\left\{y_{\sigma} ; y_{\sigma, v_{i}}=0, g_{\Delta\left(Q v_{i}\right.}\right)\left(y_{\sigma, v_{i}+1}, \ldots, y_{\sigma, n+1}\right)=0\right\} .
$$

Thus $E\left(P_{1} ; \sigma\right) \cap \cdots \cap E\left(P_{k} ; \sigma\right)=$

$$
\left\{y_{\sigma} ; y_{\sigma, \nu_{i}}=0 \text { for } i=1, \ldots, k \text { and } g_{\Delta\left(Q_{y_{k}}\right)}\left(y_{\sigma, y_{k+1}}, \ldots, y_{\sigma, n+1}\right)=0\right\}
$$

which is clearly smooth of dimension $n-k$. It is compact because $E\left(P_{1}\right) \cap \cdots \cap E\left(P_{k}\right)$ is a closed subspace of a compact divisor $E\left(P_{k}\right)$.

The divisor $E(P)$ with $\operatorname{dim} \Delta(P)=1$ is special by the following reason.

Lemma (4.8). Assume that $\operatorname{dim}(P)=1$. Then $E(P)$ has $r(\Delta(P))+1$ connected components where $r(\Delta(P))$ is the number of integral points on, $\Delta(P)-\partial \Delta(P)$.

Proof. We can find a simplex $\sigma=\left(P_{1}, \ldots, P_{n+1}\right)$ such that $P_{n}=P$ and $\Delta\left(P_{n+1}\right)$ is one of the end points $\partial \Delta(P)$. Let $f_{\Delta(P)}(z)=$ $\sum_{i=0}^{r+1} a_{i} z^{\nu_{i}}$ where $\nu_{0}, \nu_{1}, \ldots, \nu_{I+1}$ are integral points of $\Delta(P)$ in the order and $r=r(\Delta(P)) . E(P ; \sigma)$ is defined by $y_{\sigma, n}=0$ and $g_{\Delta\left(P_{n}\right)}\left(y_{\sigma, n+1}\right)=0$. As the number of the integral points on $\Delta(P)$ and on $S\left(g_{\Delta\left(P_{n}\right)}\right) \quad\left(=\right.$ the support of $\left.g_{\Delta\left(P_{n}\right)}\right)$ are equal, we may assume that $r+1$

$g_{\Delta\left(P_{n}\right)}\left(y_{\sigma, n+1}\right)=\sum_{i=0} a_{i} y_{\sigma, n+1}^{i}$. Thus by the non-degeneracy condition, $g_{\Delta\left(P_{n}\right)}\left(y_{\sigma, n+1}\right)=0$ is the disjoint union of $r+1$ planes $L(\sigma)_{i}=\left\{y_{\sigma, n+1}=\xi_{i}\right.$ and $\left.y_{\sigma, n}=0\right\}$ where $\xi_{i} \neq 0$ for $i=1, \ldots, r+1$. Let $\tau=\left(Q_{1}, \ldots, Q_{n+1}\right)$ be a simplex such that $\Delta\left(Q_{1}\right) \supset \ldots>\Delta\left(Q_{n+1}\right)$ and $Q_{i}=P$ for some $i$ and $\Delta\left(Q_{i+1}\right)=\ldots=\Delta\left(Q_{n+1}\right)=$ one point. We can find a simplex $\sigma$ as above such that $Q_{1}=P_{i_{1}}, Q_{2}=P_{i_{2}}, \ldots, Q_{i}=P_{n}$. Watching the glueing map carefully, we can see that $E(P ; \tau) \subset E(P ; \sigma)$. 
Thus $E(P)$ is covered by $E(P ; \sigma)$ where $\sigma$ is of the above type. Let $\sigma^{\prime}=\left(P_{1}^{\prime}, \ldots, P_{n+1}^{\prime}\right)$ be another simplex with $P_{n}^{\prime}=P$. Then $y_{\sigma^{\prime}}$ and $y_{\sigma}$ are identified through the matrix

$$
\Lambda=\left(\begin{array}{ccc} 
& & 0 * \\
\Lambda^{\prime} & \vdots & \vdots \\
& & \vdots \\
& & \\
* & \ldots \ldots \ldots & 1, * \\
0 & \ldots \ldots \ldots & 0, \pm-1
\end{array}\right)
$$

The sign \pm 1 means whether $P_{n+1}^{\prime}$ is on the same side of $P_{n+1}$ or not with respect to $\Delta(P)^{*}$. Thus the component $y_{\sigma^{\prime}, n+1}^{ \pm 1}=\xi_{i}$ is glued with the component $y_{\sigma, n+1}=\xi_{i}$ by $y_{\sigma, 1}=y_{\sigma^{\prime}, 1}^{\lambda_{11}} \ldots y_{\sigma^{\prime}, n-1}^{\lambda_{1, n-1}} y_{\sigma^{\prime}, n+1}^{\lambda_{1, n+1}}, \ldots$, $y_{\sigma, n-1}=y_{\sigma^{\prime}, 1}^{\lambda_{n-1,1}} \ldots y_{\sigma^{\prime}, n-1}^{\lambda n-1, n-1} y_{\sigma^{\prime}, n+1}^{\lambda} \ldots$. Thus the union of $y_{\sigma^{\prime}, n+1}^{ \pm 1}=\xi_{i} \quad$ for each $i$ make a connected component.

Remark (4.9). The above components are birationally equivalent to $P^{n-1}$

$\$ 5$. Topology of the exceptional divisors.

Let $g\left(u_{1}, \ldots, u_{n}\right)$ be a polynomial with support $S(g)$. We say that $g$ is globally non-degenerate ( $=0$-non-degenerate in [19]) if $g_{\Delta}(u)=\frac{\partial g_{\Delta}}{\partial u_{1}}(u)=\ldots=\frac{\partial g_{\Delta}}{\partial u_{n}}(u)=0$ has no solution in $\left(\mathbb{C}^{*}\right)^{n}$ for any face $\Delta$ of $\mathrm{S}(\mathrm{g}) .([15])$. In $[15]$, we have proved Theorem (5.1). Let $g$ be a globally non-degenerate polynomial. Then

(i) $\boldsymbol{x}\left(\left(\mathbb{C}^{*}\right)^{n}-g^{-1}(0)\right)=(-1)^{n} n$ ! n-dim. volume $s(g)$

(ii) If $\operatorname{dim} S(g) \geqq 3, \pi_{1}\left(\left(\mathbb{C}^{*}\right)^{n}-g^{-1}(0)\right)$ is a free abelian group of rank $n+1$. 
By the additivity of the Euler characteristics and (i), we get

Corollary (5.2). ([6], [19]). Let $g$ be as above and let $V^{*}=$ $g^{-1}(0) \cap\left(\mathbb{C}^{*}\right)^{n}$. Then $\chi\left(V^{*}\right)=(-1)^{n+1} n ! n$-dim. volume $s(g)$.

In this section, we study the topology of exceptional divisors of $\pi: \tilde{V} \longrightarrow V$ which is constructed in $\$ 4$. Let $\sigma=\left(P_{1}, \ldots, P_{k+1}\right)$ be an (unordered) k-simplex of $\Sigma^{*}$. We define $E(\sigma)=\bigcap_{i} E\left(P_{i}\right)$ and $E(\sigma)^{*}=E(\sigma)-U\{E(Q)$; $\left.Q \neq P_{1}, \ldots, P_{k+1}\right\}$. We also use the notation $E(\sigma)=E\left(P_{1}, \ldots, P_{k+1}\right)$ etc. Define $\Delta(\sigma)$ by the intersection of $\Delta\left(P_{i}\right)$. For a fixed strictly positive vertex $P$, the collection of $E\left(P, P_{1}, \ldots, P_{k}\right) *$ gives a canonical stratification of $E(P)$ where $P$ and $P_{1}, \ldots, P_{k}$ are adjacent. Let $\Delta$ be a face of $\Delta(P)$ of dimension $(n-k)$ and let $a(\Delta ; P)$ be the number of $k$-simplexes $\sigma=\left(P, P_{1}, \ldots, P_{k}\right)$ of $\Sigma^{*}$ on the closure $\left(\Delta^{*}\right)$.

The main result of this section is

Theorem (5.3). (i) Assume that $\sigma=\left(P, P_{1}, \ldots, P_{k}\right)$ be a $k$-simplex of $\Sigma^{*}$. The Euler characteristic $X\left(E(\sigma)^{*}\right)$ is non-zero if and only if $\operatorname{dim} \Delta(\sigma)=$ $n-k$. Let $\tau=\left(Q_{1}, \ldots, Q_{n+1}\right)$ be an $n$-simplex such that $\left\{P, P_{1}, \ldots, P_{k}\right\}=$ $\left\{Q_{1}, \ldots, Q_{k+1}\right\}$. Then $\chi(E(\sigma) *)=(-1)^{n-k+1}(n-k)$ ! volume $s\left(g_{\Delta(\sigma)}\left(y_{\tau, k+2}, \ldots, y_{\tau, n+1}\right)\right)$.

(ii) The isomorphism class of $E(\sigma) *$ depends only on $\Delta(\sigma)$ if $\operatorname{dim} \Delta(\sigma)=$ $n-k$. It does not/depend on the particular choice of $\Sigma^{*}$ either. (iii) Let $\Delta$ be a $(n-k)$-dimensional face of $\Delta(P)$ and let $X(\Delta ; P)$ be $X\left(E(\sigma)^{*}\right)$ where $\sigma$ is a k-simplex on the closure $\left(\Delta^{*}\right)$ with $P \in \sigma$. Then $\chi(E(P))=\Sigma a(\Delta ; P) X(\Delta ; P)$ where the sum is taken for every face of $\Delta(P)$.

Corollary (5.4). (i) The Euler characteristic of the top dimensional stratum $E(P) *$ is $(-1)^{n+1}(n+1)$-dim. volume $C(0, \Delta(P))$ where $C(0, \Delta(P))$ is the cone of $\Delta(P)$ with the origin. $/ d(P)$ 
(ii) The birational class of the exceptional divisor $E(P)$ depends only on $\Delta(P)$. If $\operatorname{dim} \Delta(P)=r<n$, there exists a compact complex manifold $M(P)$ of dimension $I-1$ such that $E(P)$ is birationally equivalent to $P^{n-r} \times M(P)$.

The proof of Theorem (5.3) and Corollary (5.4) occupies the rest of of this section.

Let $\sigma=\left(P_{1}, \ldots, P_{n+1}\right)$ be a simplex with $\Delta\left(P_{1}\right)>\ldots>\Delta\left(P_{n+1}\right)$. By the definition, ${ }\left(P_{i_{1}}, \ldots, P_{i_{k}}\right) * \subset \mathbb{C}_{\sigma}^{n+1}$ and $E\left(P_{i_{1}}, \ldots, P_{i_{k}}\right) *$ is defined by $y_{\sigma, i_{1}}=\ldots=y_{\sigma, i_{k}}=0$ and $g_{\Delta\left(P_{i_{k}}\right)}\left(y_{\sigma, i_{k+1}}, \ldots, y_{\sigma, n+1}\right)=0$ with $y_{\sigma, j} \neq 0$ for $j \neq i_{1}, \ldots, i_{k}$. Here we have assumed that $i_{1}<i_{2}<\ldots<i_{k}$. The polynomial $g_{\Delta\left(P_{i_{k}}\right)}$ is defined by $f_{\Delta\left(P_{i_{k}}\right)}\left(\pi_{\sigma}\left(y_{\sigma}\right)\right)=\left\{\prod_{i=1}^{n+1} y_{\sigma, i} d\left(P_{i}\right)\right\} g_{\Delta\left(P_{i_{k}}\right)}$. Thus it is easy to see that $g_{\Delta\left(P_{i_{k}}\right)}$ is globally non-degenerate because $f$ is non-degenerate on $\Delta\left(P_{i_{k}}\right)$. (Compare with Lemma (5.2) of $\left[\begin{array}{ll}15 & \text { ]). As }\end{array}\right.$ $\operatorname{dims}\left(g_{\Delta\left(P_{i_{k}}\right)}\right)=\operatorname{dim} \Delta\left(P_{i_{k}}\right)$, the assertion (i) of Theorem (5.3) is immediate from Corollary (5.2). Assume that $P=P_{1}$ and $\operatorname{dim} \Delta(P)=n$. Then $E(P) *$ is defined by $g_{\Delta(P)}\left(y_{\sigma, 2}, \ldots, y_{\sigma, n+1}\right)=0$ where $f_{\Delta(P)}\left(\pi_{\sigma}\left(y_{\sigma}\right)\right)=$ $y_{\sigma, 1}^{d\left(P_{1}\right)} \ldots y_{\sigma, n+1}^{d\left(P_{n+1}\right)} g_{\Delta(P)}\left(y_{\sigma, 2}, \ldots, y_{\sigma, n+1}\right)$. Thus we have the equalities $(n+1)$ ! volume $C(0, \Delta(P))=(n+1)$ ! volume $C\left(0, S\left(\pi_{\sigma^{*} f}(P)\right)\right)$
$=(n+1)$ ! volume $S\left(g_{\Delta(P)}\right) \cdot \frac{d(P)}{n+1}$
$=n !$ volume $S\left(g_{\Delta(P)}\right) \cdot d(P)$. 
This proves the assertion (i) of Corollary (5.4).

Let $\Sigma^{* 1}$ be another simplicial subdivision of $\Gamma^{*}(f)$ and let $\pi^{\prime}: \widetilde{V}^{\prime} \rightarrow V$ be the associated resolution. We denote the exceptional divisors in $\pi^{\prime}: \widetilde{V}^{\prime} \rightarrow V$ by $E^{\prime}(P), E^{\prime}(\sigma)$ etc. Let $\sigma=\left(P_{1}, \ldots, P_{n+1}\right)$ be a simplex of $\Sigma^{*}$ and let $\sigma^{\prime}=\left(P_{1}, \ldots, P_{n+1}^{\prime}\right)$ be a simplex of $\Sigma^{* 1}$. We assume that

$(5.5)$

$$
\left\{\begin{array}{l}
\Delta\left(P_{1}\right)>\ldots>\Delta\left(P_{n+1}\right) \\
\Delta\left(P_{1}^{\prime}\right)>\ldots>\Delta\left(P_{n+1}^{\prime}\right)
\end{array} \text { and } \Delta\left(P_{k}\right)=\Delta\left(P_{k}\right)\right.
$$$$
\text { and } \operatorname{dim} \Delta\left(P_{k}\right)=n+1-k \quad \text { for some } k \text {. }
$$

$E\left(P_{1}, \ldots, P_{k}\right)$ is defined in $\mathbb{C}_{\sigma}^{n+1}$ by

$$
y_{\sigma, 1}=\ldots=y_{\sigma, k}=0 \text { and } g_{\Delta_{k}}\left(y_{\sigma, k+1}, \ldots, y_{\sigma, n+1}\right)=0
$$

where $\Delta_{k}=\Delta\left(P_{k}\right)$ and $f_{\Delta_{k}}\left(\pi_{\sigma}\left(y_{\sigma}\right)\right)=\prod_{y_{\sigma, i}}^{d\left(P_{i}\right)} \cdot g_{\Delta_{k}}\left(y_{\sigma, k+1}, \ldots, y_{\sigma, n+1}\right)$. $E^{\prime}\left(P_{i}^{\prime}, \ldots, P_{k}^{\prime}\right)$ is defined in $\mathbb{C}_{\sigma^{\prime}}^{n+1}$ by

$$
y_{\sigma^{\prime}, 1}=\ldots=y_{\sigma^{\prime}, k}=0 \text { and } g_{\Delta_{k}}^{\prime}\left(y_{\sigma^{\prime}, k+1}, \ldots, y_{\sigma^{\prime}, n+1}\right)=0
$$

where $\left.f_{\Delta_{k}}\left(\pi_{\sigma^{\prime}}\left(y_{\sigma^{\prime}}\right)\right)=\prod_{\sigma^{\prime}, i}^{d\left(P^{\prime}\right.}\right) g_{\Delta_{k}^{\prime}}\left(y_{\sigma^{\prime}, k+1}, \ldots, y_{\sigma^{\prime}, n+1}\right)$.

By the assumption that $\Delta\left(P_{k}\right)=\Delta\left(P_{k}\right)$ and $\operatorname{dim} \Delta\left(P_{k}\right)=n+1-k$, Z-submodules generated by $P_{1}, \ldots, P_{k}$ and $P_{1}, \ldots, P_{k}^{\prime}$ are equal and they are equal to the submodules generated by the integral points of Therefore the matrix $\sigma^{-1} \cdot \sigma^{\prime}$ can be written as $\operatorname{closure}\left(\Delta_{\mathrm{k}}^{*}\right)$.

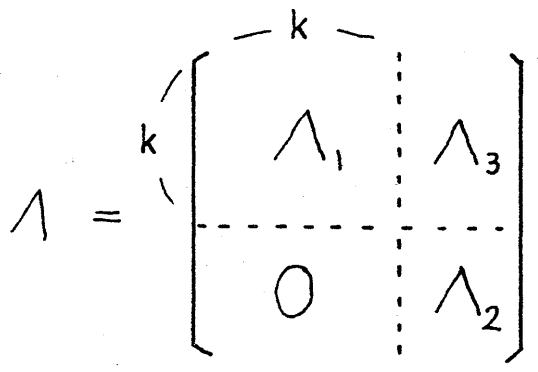


where $\Lambda_{i}(i=1,2)$ are unimodular. Write $\mathbb{C}_{\sigma}^{n+1}$ as $\mathbb{C}_{\sigma}^{k} \times \mathbb{C}_{\sigma}^{n+1-k}$ and $y_{\sigma}=\left(y_{1}, y_{2}\right)$ where $y_{1} \in \mathbb{C}_{\sigma}^{k}$ and $y_{2} \in \mathbb{C}_{\sigma}^{n+1-k} \cdot y_{2}$ is the coordinate of the subspace $\mathbb{C}_{\sigma}^{n+1-k}$ where $E\left(P_{1}, \ldots, P_{k}\right)$ are defined by $g_{\Delta_{k}}\left(y_{2}\right)=0$. Respectively we write $c_{\sigma^{\prime}}^{n+1}$ as $\mathbb{C}_{\sigma^{\prime}}^{k} \times \mathbb{C}_{\sigma^{\prime}}^{n+1-k}$ and $y_{\sigma^{\prime}}=\left(y_{1}^{\prime}, y_{2}^{\prime}\right)$. Now we define a birational mapping $\pi_{\Lambda_{2}}: \mathbb{C}_{\sigma^{\prime}}^{n+1-k} \rightarrow \mathbb{C}_{\sigma}^{n+1-k}$ by $y_{2}=\left(y_{2}^{\prime}\right)^{\Lambda_{2}}$. we will show that $\pi_{\Lambda_{2}}$ induces a desired biholomorphic mapping from $E^{\prime}\left(P_{1}^{\prime}, \ldots, P_{k}^{\prime}\right)^{*}$ to $E\left(P_{1}, \ldots, P_{k}\right)^{*}$. By the definition of $\Lambda$, we have $y_{\sigma}=\left(y_{\sigma^{\prime}}\right)^{\Lambda}$. In particular, $y_{2}=y_{2}^{\prime} \Lambda_{2}$. Thus we have $f_{\Delta_{k}}\left(y_{\sigma^{\prime}} \hat{)}\right)=f_{\Delta_{k}}\left(\pi_{\sigma^{\prime}}\left(y_{\sigma^{\prime}}\right)\right)$ which implies that

$g_{\Delta_{k}}\left(y_{2}^{\prime} \Lambda_{2}\right)=g_{\Delta_{k}}^{\prime}\left(y_{2}^{\prime}\right) \cdot \prod_{i=k+1}^{n+1}\left(y_{\sigma^{\prime}, i}\right)^{\alpha_{i}}$ for some $\alpha_{k+1}, \ldots, \alpha_{n+1} \in \mathbb{Z}$. The last equality says that $\pi_{1_{2}}\left(E^{\prime}\left(P_{1}^{\prime}, \ldots, P_{k}^{\prime}\right)^{*}\right)=E\left(P_{1}, \ldots, P_{k}\right)^{*}$. This completes the proof of the assertion (ii) of Theorem (5.3). The assertion (iii) of Theorem (5.3) is immediate from (ii) and the additivity of the Euler characteristics.

To prove (ii) of Corollary (5.4), we take a strictly positive vertex $P$ such that $\operatorname{dim} \Delta(P)=r$ and $0<r<n$. Let $\sigma=\left(P_{1}, \ldots, P_{n+1}\right)$ be a simplex with $P_{n-r+1}=P$ and $\Delta\left(P_{1}\right)>\ldots>\Delta\left(P_{n+1}\right) \cdot E(P, \sigma)$ is the product $\mathbb{C}_{\sigma}^{n-r} \times\left\{E\left(P_{1}, \ldots, P_{n-r+1}\right) \cap \mathbb{C}_{\sigma}^{n+1}\right\}$. Thus we can take $E\left(P_{1}, \ldots, P_{n-r+1}\right)$ as $M(P)$. The isomorphism $E(P ; \sigma) \rightarrow \mathbb{C}_{\sigma}^{n-r_{X}}\left(E\left(P_{1}, \ldots, P_{n-r+1}\right) \cap\right.$ $\mathbb{C}_{\sigma}^{n+1}$ ) extends to a desired birational mapping. This completes the proof. Corollary (5.6). Let $P$ be a strictly positive vertex with $\operatorname{dim} \Delta(P)=1$. Then each component is birationally equivalent to $P^{n-1}$ and the Euler 
characteristic is equal to $a_{n}=$ the number of $(n-1)$ - simplexes $\sigma^{\prime}=$ $\left(P_{1}, \ldots, P_{n}\right)$ such that $P_{n}=P$ and $\Delta\left(P_{1}\right)>\ldots \supset \Delta\left(P_{n}\right)$.

Remark (5.7). Two simplexes with the same vertices are counted once.

\$6. The surface singularity.

(good, In this section, we study the case $n=2$ in detail. Let $\pi: \widetilde{v} \longrightarrow v$ be aresolution of $V$ which is constructed in $\$ 4$. Let $E_{i}(i=1, \ldots, k)$ be the irreducible components of $\pi^{-1}(0)$. The resolution graph $\Gamma$ is defined in the following way. For any $E_{i}$, we associate a vertex $v_{i}$ with weight $m_{i}$ which is the self-intersection number of $E_{i}$. When $E_{i}$ is not a rational curve, we also put the genus $g_{i}$ of $E_{i}$ to $v_{i}$. If $E_{i} \cap E_{j} \neq \varnothing, v_{i}$ and $v_{j}$ are joined by a line segment. A lot of information about the resolution is derived from this weighted graph $\Gamma \cdot([13],[4],[3],[7]$.

The 2-skeleton $S_{2} \Gamma^{*}(f)$ can be expressed by a graph through a hyperplane section. (See Figure (2.1) in \$2.) we will show that $\Gamma$ is obtained from $S_{2} \Gamma^{*}(f)$ by a canonical surgery. For a 2-dimensional face $\Delta$ of $\Gamma(f)$, we define an integer $g(\Delta)$ by the number of the integral points on $\Delta-\partial \Delta$. Similarly for a l-dimensional face $\Xi$ of $\Gamma(f)$, we define $r(\Xi)$ by the number of integral points on $\Xi-\partial Z$. For any line segment $\overline{P Q}$ of $\Gamma^{*}(f), r(P, Q)$ is defined by $r(\Delta)$ where $\Delta^{*}$ is the interior of $\overline{P Q}$. Our main result in this section is

Theorem (6.1). Let $\pi: \widetilde{v} \rightarrow \vee$ be the resolution associated with $\Sigma^{*}$. Then we have

(i) If $P$ is strictly positive and $\operatorname{dim} \Delta(P)=2, E(P)$ has genus $g(\Delta(P))$.

(ii) If $P$ is strictly positive and $\operatorname{dim} \Delta(P)=1, E(P)$ is $r(\Delta(P))+1$ disjoint copies of rational curves.

Assume that $\Sigma^{*}$ is canonical $\frac{\text { in the sense of }(3.5) .}{T h e n}$

(iii) the resolution graph is obtained by the following surgery of $S_{2}\left(\Gamma^{*}(f)\right)$. 
Let $\overline{P Q}$ be a line segment of $S_{2} \Gamma^{*}(f)$ and assume that $P$ is strictly positive. Let $C=\operatorname{det}(P, Q)$. If $C=1$, we put $r(P, Q)+1$ copies of rational curves with self-intersection -1 between $P$ and $Q$ as follows:
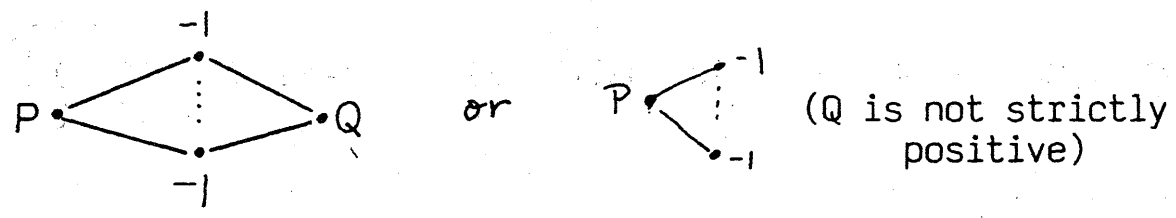

If $c>1$, let $c_{1}$ be the unique integer such that $P_{1}=\frac{1}{c} Q+\frac{c_{1}}{c} P$ is integral and $0<c_{1}<c$. Let $\frac{c}{c_{1}}=\left[m_{1}, \ldots, m_{k}\right]$. We insert $r(p, q)+1$ copies of a chain of rational curves. $-m_{1}-m_{2} \ldots-m_{k}$ between $P$ and $Q$. Those vertices which are not strictly positive are omitted after the surgery from the resolution graph.

(iv) Let $P$ be a strictly positive vertex with $\operatorname{dim} \Delta(P)=2$. Let $Q_{1}, \ldots, Q_{S}$ be the vertices of $\Sigma^{*}$ which are adjacent to $P$ and $\operatorname{dim} \Delta\left(Q_{i}\right)=1$. Let $P=\left(\begin{array}{l}p_{1} \\ p_{2} \\ p_{3}\end{array}\right)$ and $Q_{i}=\left(\begin{array}{l}q_{1 i} \\ q_{2 i} \\ q_{3 i}\end{array}\right)$. Then the self-intersection number of $E(P)$ is

$$
-\frac{\sum_{i=1}^{s}\left(r\left(P, Q_{i}\right)+1\right) q_{1, i}}{p_{1}}
$$

Before the proof of Theorem (6.1), we explain the theorem by two examples. Example (6.2). Let $f\left(z_{1}, z_{2}, z_{3}\right)=z_{1}^{2}+z_{2}^{3}+z_{3}^{5}$. Then $\mathrm{S}_{2} \Gamma^{*}(\mathrm{f})$ is as follows. (o implies "not strictly positive.")

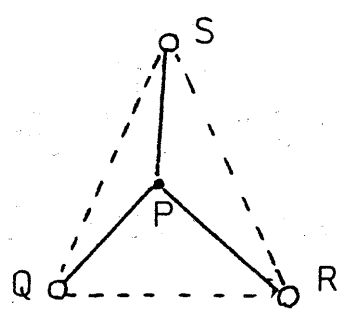

$$
\begin{aligned}
& P=t(15,10,6), Q={ }^{t}(1,0,0) \\
& R={ }^{t}(0,1,0), S={ }^{t}(0,0,1)
\end{aligned}
$$


It is easy to see that $g(\Delta(P))=0 . \operatorname{det}(P, Q)=2$. $Q^{\prime}=\frac{1}{2}\left(\begin{array}{l}1 \\ 0 \\ 0\end{array}\right]+\frac{1}{2}\left[\begin{array}{l}15 \\ 10 \\ 6\end{array}\right]=\left[\begin{array}{l}8 \\ 5 \\ 3\end{array}\right]$. As $\operatorname{det}(P, R)=3$ and $R^{\prime}=\frac{1}{3}\left[\begin{array}{l}0 \\ 1 \\ 0\end{array}\right]+\frac{2}{3}\left[\begin{array}{l}15 \\ 10 \\ 6\end{array}\right]=\left[\begin{array}{l}10 \\ 7 \\ 4\end{array}\right.$ we have $\frac{3}{2}=2-\frac{1}{2}$. As $\operatorname{det}(P, S)=5$ and $S^{\prime}=\frac{1}{5}\left[\begin{array}{l}0 \\ 0 \\ 1\end{array}\right]+\frac{4}{5}\left[\begin{array}{l}15 \\ 10 \\ 6\end{array}\right]=\left[\begin{array}{c}12 \\ 8 \\ 5\end{array}\right]$, we have $\frac{5}{4}=[2,2,2,2]$. As $r(P, Q)=r(P, R)=r(P, S)=0$, $E_{p}^{2}=-\frac{8+10+12}{15}=-2$. Thus the resolution graph is

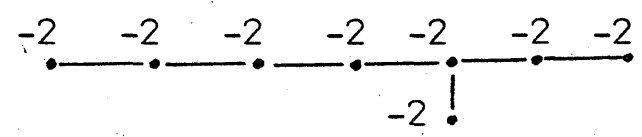

Example (6.3). Let $f\left(z_{1}, z_{2}, z_{3}\right)=z_{1}^{3}+z_{2}^{4}+z_{3}^{4}+z_{1} z_{2} z_{3} \cdot \Gamma(f)$ has three 2-dimensional faces with genus $0 . S_{2} \Gamma^{*(f)}$ is:

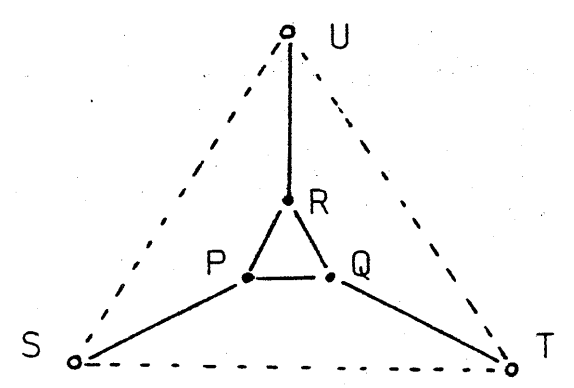

$$
\begin{aligned}
& P={ }^{t}(2,1,1), S={ }^{t}(1,0,0) \\
& Q={ }^{t}(4,5,3), T={ }^{t}(0,1,0) \\
& R={ }^{t}(4,3,5), U={ }^{t}(0,0,1)
\end{aligned}
$$

(1) $\operatorname{det}(P, S)=1, \quad r(P, S)=3$. Thus we add 4 vertices of self-intersection number -1 to $P$.

(2) $\operatorname{det}(P, Q)=2, \quad r(P, Q)=0$. As $\frac{1}{2} P+\frac{1}{2} Q=\left(\begin{array}{l}3 \\ 3 \\ 2\end{array}\right), \quad c_{1}=1$. Thus we add -2 between $P$ and $Q$.

(3) $\operatorname{det}(Q, T)=1, \quad r(Q, T)=0$. Thus we add -1 to $Q$.

(4) $\operatorname{det}(Q, R)=8, \quad r(Q, R)=0$. As $\frac{1}{8} Q+\frac{1}{8} R=\left(\begin{array}{l}1 \\ 1 \\ 1\end{array}\right)$, we add - between $Q$ and $R \cdot \overline{P R}$ and $\overline{R U}$ are similar with $\overline{P Q}$ and $\overline{Q T}$. 
$E(P)^{2}=-\frac{4 \cdot 3+3+3}{2}=-9$

$E(Q)^{2}=-\frac{3+4+1}{4}=-2$

$E(R)^{2}=-\frac{1+3+4}{4}=-2$.

Thus the resolution graph is

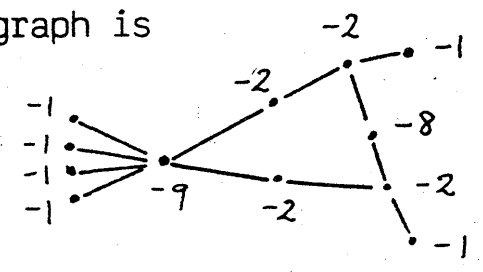

which is blown down to

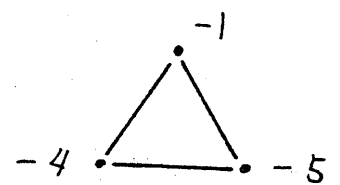

Now we are ready to prove Theorem $(6.1)$. Let $P$ be a strictly positive vertex of $\Sigma^{*}$ with $\operatorname{dim} \Delta(P)=1$. Then $P$ is on the line segment $\Delta(P)^{*}$. Thus there are exactly two vertices $P_{1}, P_{i}$ on the closure of $\Delta(P) *$ which are adjacent to $P$.

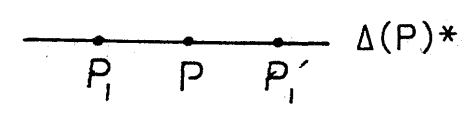

By Corollary (5.6), we have $X(E(P))=(r(\Delta(P))+1) \cdot 2$. As $E(P)$, has $r(\Delta(P)+1)$ components which are birationally equivalent, each component must be a rational curve $P^{1}$. We will see this directly. Let $\sigma=\left(P_{1}, P, P_{2}\right)$ and $\sigma^{\prime}=\left(P_{1}^{\prime}, P, P_{2}^{\prime}\right)$ be two simplexes where $P_{2}$ and $P_{2}^{\prime}$ are taken on the same side with respect to $\Delta(P) *$ for brevity's 
sake. In $\mathbb{a}_{\sigma}^{3}, E(P ; \sigma)$ is defined by $y_{\sigma, 2}=0$ and $y_{\sigma, 3}=\xi_{i}$ $(i=1, \ldots, r(\Delta(P))+1)$. In $\mathbb{C}_{\sigma^{\prime}}^{3}, E\left(P ; \sigma^{\prime}\right)$ is defined by $y \sigma^{\prime}, 2=0$ and $y_{\sigma, 3}=\xi_{i}$. They are glued by the matrix

$$
\sigma^{-1} \cdot \sigma^{\prime}=\left(\begin{array}{ccc}
-1 & 0 & b \\
a & 1 & c \\
0 & 0 & 1
\end{array}\right)
$$

Thus $y_{\sigma, 3}=y_{\sigma^{\prime}, 3}$ and $y_{\sigma, 1}=y_{\sigma^{\prime}, 1}^{-1} \cdot y_{\sigma^{\prime}, 3}^{b}$. Namely on each component $y_{\sigma, 3}=y_{\sigma^{\prime}, 3}=\xi_{i}, y_{\sigma, 1}=y_{\sigma^{\prime}, 1}^{-1} \cdot \xi_{i}^{b}$. Thus it is a rational curve. This proves (ii) of Theorem (6.1).

To prove the assertion ( $i$ ) of the theorem, we need

Lemma (6.4). Let $\Delta$ be a compact convex polyhedron in $\mathbb{R}^{2}$ with integral vertices $P_{1}, \ldots, P_{k}$. Then 2 volume $\Delta=2 g(\Delta)+\sum_{i=1}^{k}\left(r\left(\Delta_{i}\right)+1\right)-2$, where $\partial \Delta=\Delta_{1} U \Delta_{2} \quad \ldots U_{\Delta_{k}}$.

Proof. Step 1. Assume that $k=3, g(\Delta)=r\left(\Delta_{1}\right)=r\left(\Delta_{2}\right)=r\left(\Delta_{3}\right)=0$. By a parallel translation if necessary, we may assume that $P_{1}=\left(\begin{array}{l}0 \\ 0\end{array}\right)$. Then $P_{2}$ and $P_{3}$ are primitive vectors and $2 \cdot \operatorname{volume} \Delta=\operatorname{det}\left(P_{2}, P_{3}\right)$.

Assume that $c=\operatorname{det}\left(P_{2}, P_{3}\right)>1$. By Lemma (3.3), we can find $0<c_{1}<c$ such that $Q=\frac{1}{c} P_{2}+\frac{C_{1}}{c} P_{1}$ is integral. However this is a contradiction to the assumption $g(\Delta)=r\left(\Delta_{1}\right)=0$ as $Q \in \Delta-\left\{P_{1}, P_{2}, P_{3}\right\}$. Thus the assertion is true in this case.

Step 2. $k=3$. Assume that $r\left(\Delta_{1}\right) \neq 0$ or $g(\Delta) \neq 0$. Then we divide $\Delta$ into two or three triangles respectively. 

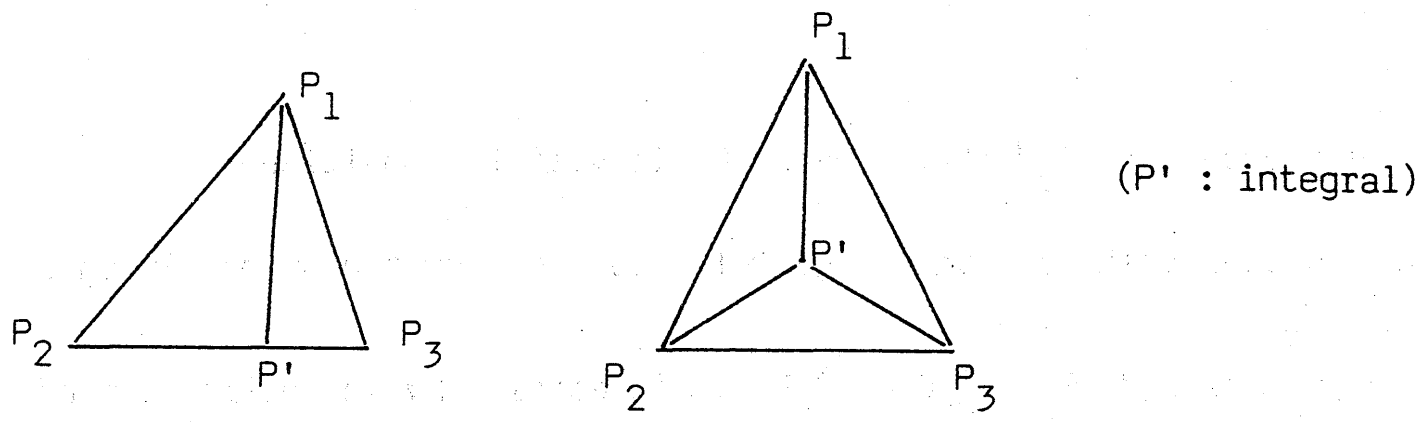

It is easy to see that both sides of the assertion are additive under the above divisions. Therefore this case is reduced to Step 1 by a finite subdivision.

Step 3. The assertion for general $k \geqq 3$ is proved by the induction on $k$ and the additivity of the assertion under the following division:

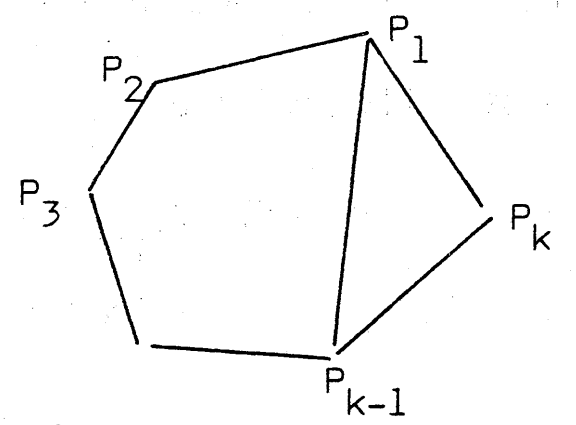

Let $P$ be a strictly positive vertex of $\Sigma^{*}$ such that $\operatorname{dim} \Delta(P)=2$. Let $\Delta_{1}, \ldots, \Delta_{k}$ be the boundaries of $\Delta(P)$. Then $P$ has $k$-branches in $\Gamma^{*}(f)$ which correspond bijectively to $\Delta_{i}(i=1, \ldots, k)$. Each branch has a unique vertex $P_{i}$ which is adjacent to $P$ in $\Sigma^{*}$. Let $\sigma=\left(P, P_{1}, P_{1}^{\prime}\right)$ be a simplex. In $\mathbb{C}_{\sigma}^{3}, E(P ; \sigma)$ is defined by $y_{\sigma, 1}=0$, $g_{\Delta(P)}\left(y_{\sigma, 2}, y_{\sigma, 3}\right)=0$. By Theorem (5.3), we have $X(E(P))=-2 \operatorname{volume}\left(S\left(g_{\Delta(P)}\right)\right)+$ $\sum_{i=1}^{k}\left(r\left(\Delta_{i}\right)+1\right)$. We use the equality $g(\Delta(P))=g\left(S\left(g_{\Delta(P)}\right)\right)$ which is immediate from the fact that $\sigma$ is a unimodular matrix. Thus by Lemma $(6.4)$, we get

$$
X(E(P))=-2 g(\Delta(P))+2
$$

which implies that $g(\Delta(P))$ is the genus of $E(P)$.

Now we assume that $\Sigma^{*}$ is a canonical simplicial subdivision. Let 
$\overline{P Q}$ be a line segment of $S_{2} \Gamma^{*}(f)$ where $P$ is strictly positive.

Case 1. Assume that $c=\operatorname{det}(P, Q)>1$. Let $c_{1}$ be the unique integer such that $O<c_{1}<c$ and $P_{1}=\frac{1}{C} Q+\frac{C_{1}}{c} P$ is integral. By the definition of $\Sigma^{*}, \Sigma^{*}$ has $k$ vertices $P_{1}, \ldots, P_{k}$ on $\overline{P Q}$ which are inductively defined by

$$
P_{i+1}=\frac{1}{c_{i}} Q+\frac{c_{i+1}}{c_{i}} P_{i} \quad(i=0, \ldots, k-1)
$$

where $P_{0}=P, \quad 0<c_{i+1}<c_{i}$ for $i=0, \ldots, k-1$ and $c_{0}=c$ and $c_{k}=1$. Observe that $P_{1}, \ldots, P_{k}$ are strictly positive. Let $\sigma_{i}=\left(P_{i}, P_{i+1}, R_{i}\right)$ be a simplex of $\Sigma^{*}$. In $\mathbb{C}_{\sigma_{i}}^{3}, E\left(P_{i}\right)$ is defined by $y_{\sigma_{i}, 1}=0$ and $y_{\sigma_{i}, 3}=\xi_{j}(j=1, \ldots, r(P, Q)+1)$ and $E\left(P_{i+1}\right)$ is defined by $y_{\sigma_{i}, 2}=0$ $y_{\sigma_{i}, 3}=\xi_{j}(j=1, \ldots, r(P, Q)+1)$. Thus each component of $E\left(P_{i}\right)$ has a nonempty intersection with only one component of $E\left(P_{i+1}\right)$. We note the components of $E\left(P_{i}\right)$ by $E_{i, j}(j=1, \ldots, r(P, Q)+1)$. We may assume that $E_{i, j} \cdot E_{i, k}=0(j \neq k)$ and $E_{i, j} \cdot E_{i+1, j}=E_{i, j} \cdot E_{i-1, j}=1, E_{i, j} \cdot E_{i+1, k}=$ $E_{i, j} \cdot E_{i-1, k}=0(j \neq k)$. To compute $E_{i, j}^{2}$, we consider the divisor of thelomorphic function $\pi^{*} z_{1}$. In $\mathbb{a}_{\sigma_{i}}^{3}, \pi^{*} z_{1}=$

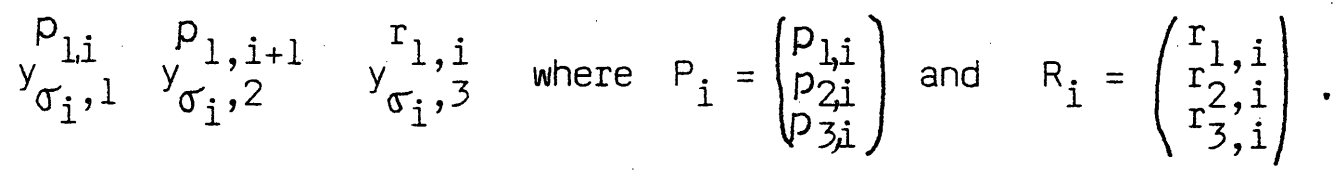


Thus $\left(\pi^{*} z_{1}\right)=\sum_{i=0}^{k+1} p_{1 i} E\left(P_{i}\right)+D$ where $D$ has no intersection with any $E\left(P_{i}\right)$ $(i=1, \ldots, k)$. By Theorem 2.6 of $[8],\left(\pi^{*} z_{1}\right) \cdot E_{i j}=0$. Thus we get $-E_{i j}^{2}=\frac{p_{1, i-1}+p_{1, i+1}}{p_{1, i}}=m_{i}$ by Lemma (3.6) where $\frac{c}{c_{1}}=\left[m_{1}, \ldots, m_{k}\right]$.

Case 2. Assume that $c=\operatorname{det}(P, Q)=1$. By the definition of the canonical primitive sequence, $\Sigma^{*}$ has a unique vertex $P_{1}=P+Q$ on $\overline{P Q}$. Let $E_{1}, \ldots, E_{r(P, Q)+1}$ be the components of $E\left(P_{1}\right)$. Again by Theorem (2.6) of [ 8 ], we get $E_{i}^{2}=-1$. This proves the assertion (iii) of Theorem (6.1). The assertion (iv) is proved by the same argument. This completes the proof of Theorem (6.1).

In practice, the following is useful for the computation of $g(\Delta)$.

Corollary (6.5). Let $P$ be a strictly positive vertex of $\sum^{*}$ with $\operatorname{dim} \Delta(P)=2$. Then $2-2 g(\Delta(P))=-\frac{6}{d(P)}$ volume $c(0, \Delta(P))+\sum_{i=1}^{k}\left(r\left(\Delta_{i}\right)+1\right)$ where $\partial \Delta(P)=\Delta_{l} \cup \ldots \cup \Delta_{k}$.

Now we study the resolutions of several interesting singularities. (A). Weighted homogeneous polynomials.

The surface singularities with $\mathbb{C}^{*}$-action were studied by Orlik-wagreich in $[17]$. Our method gives an alternative description of the continuous fractions which were derived in $[17]$ from the orbit invariants of the $C^{*}$ action.

(I). Pham-Brieskorn variety. 
Let $f_{a}(z)=z_{1}^{a_{1}}+z_{2}^{a_{2}}+z_{3}^{a_{3}}$ where $a_{i} \geqq 2$. Let $s=g . c \cdot d \cdot\left(a_{1}, a_{2}, a_{3}\right)$ and let $s s_{i}=$ g.c.d. $\left(a_{i-1}, a_{i+1}\right)$ modulo 3 . Then $s_{1}, s_{2}, s_{3}$ are mutually coprime and we can write $a_{i}=s s_{i-1} s_{i+1} a_{i}^{\prime}$ modulo 3 for $i=1,2,3$ where $a_{1}^{\prime}, a_{2}^{\prime}, a_{3}^{\prime}$ are mutually coprime. The dual Newton diagram $\Gamma^{*}(f)$ is

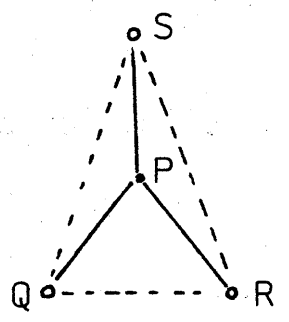

$$
\begin{aligned}
& P=\left(\begin{array}{lll}
s_{1} & a & a_{3}^{\prime} \\
s_{2} & a_{1}^{\prime} & a_{3} \\
s_{3} & a_{1} & a_{2}^{\prime}
\end{array}\right), Q=\left(\begin{array}{l}
1 \\
0 \\
0
\end{array}\right), R=\left(\begin{array}{l}
0 \\
1 \\
0
\end{array}\right) \\
& S=\left(\begin{array}{l}
0 \\
0 \\
1
\end{array}\right)
\end{aligned}
$$

Surgery data are

\begin{tabular}{|c|c|c|c|}
\hline & $c$ & $c_{1}$ & $r+1$ \\
\hline$\overline{P Q}$ & $c_{Q}=a_{1}^{\prime}$ & $c_{Q, 1} P_{1}+1 \equiv 0\left(c_{Q}\right)$ & $s_{1}$ \\
\hline$\overline{P R}$ & $c_{R}=a_{2}^{\prime}$ & $c_{R, 1} P_{2}+1 \equiv 0\left(c_{R}\right)$ & $s_{2}$ \\
\hline$\overline{P S}$ & $c_{S}=a_{3}^{\prime}$ & $c_{S, 1} P_{3}+1 \equiv 0\left(c_{S}\right)$ & $s_{3}$ \\
\hline
\end{tabular}

$$
g(E(P))=\frac{1}{2} s\left\{s s_{1} s_{2} s_{3}-\left(s_{1}+s_{2}+s_{3}\right)\right\}+1 .
$$

We assume that $s_{1} \leqq s_{2} \leqq s_{3}$. Then it is easy to see that $g(E(P))=0$ if and only if

$$
\begin{aligned}
& \left(\#_{1}\right) \quad s=s_{1}=s_{2}=1 \quad \text { or } \\
& \left(\#_{2}\right) \quad s=2, s_{1}=s_{2}=s_{3}=1 \quad \text {. }
\end{aligned}
$$

Note that $\left(\#_{1}\right)$ is equivalent to g.c.d. $\left(a_{3}, a_{1}\right)=$ g.c.d. $\left(a_{3}, a_{2}\right)=1$ and $\left(\#_{2}\right)$ is equivalent to g.c.d. $\left(a_{i}, a_{j}\right)=2$ for any $i \neq j$. 
Let $k_{a}=f_{a}^{-1}(0) \cap S^{5}$. $k_{a}$ is diffeomorphic to the boundary of a tubular neighborhood of the total exceptional divisors. $g(E(P))=0$ is equivalent to $H_{*}\left(K_{a} ; Q\right)=H_{*}\left(S^{3} ; Q\right)([\&])$.

As a special case of $(i), H_{*}\left(K_{a} ; \mathbb{Z}\right)=H_{*}\left(S^{3} ; \mathbb{Z}\right)$ if and only if $\left(\#_{1}\right)^{\prime}$ $\left(a_{i}, a_{j}\right)=1$ for $i \neq j$ by $[2]$.

In general, different polynomials may have the same resolution graph. ([11], [7]). For example, Pham-Brieskorn polynomials $x^{2}+y^{57}+z^{114}, x^{3}+y^{29}+z^{87}$, $x^{8}+y^{9}+z^{72}$ have the same minimal resolution: $\underbrace{28}_{-1}$ (28 is the genus.) However we have

Theorem (6.6). Assume that $K_{a}$ is a Q-homology sphere. Then

(i) There is no other polynomial $f_{b}$ which has the same resolution graph as fa:

(ii) The fundamental group $\pi_{1}\left(k_{a}\right)$ is a central extension of a Dyck (or a triangle) group.

Proof. If $a=\left(2,2, a_{3}\right)$, the resolution is $A_{a_{3}-1}$. Thus we can assume that $a \neq\left(2,2, a_{3}\right)$ i.e. $f_{a}$ is not of $A_{k}$ type. We assume that $a_{1}^{\prime} \leqq a_{2}^{\prime} \leqq a_{3}^{\prime}$. First observe that the intersection number $E(P)^{2}$ (after blowing down exceptional divisors of the first kind) is equal to

$(*)-\left\{\frac{c_{Q, 1}}{c_{Q}} s s_{1}+\frac{c_{R, 1}}{c_{R}} s s_{2}+\frac{c_{S, 1}}{c_{S}} s s_{3}+\frac{s}{a_{1}^{\prime} a_{2}^{\prime} a_{3}^{\prime}}\right\}$. (Here $c_{Q, 1}=0$ if $c_{Q}=1$. )

Case 1. $a_{1}>1$. Then the resolution graph has three distinct families 
of branches which correspond to the continuous fractions $c_{Q} / c_{Q, I}, c_{R} / c_{R, I}$, $c_{s} / c_{S, 1}$. Thus the resolution graph determines $a_{1}^{\prime}, a_{2}^{\prime}, a_{3}^{\prime}, s s_{1}, s s_{2}, s s_{3}$. Therefore $s=$ g.c.d.(ss $\left.s_{1} s s_{2}, s s_{3}\right)$ and $s_{i}$ are also determined. Thus $a=$ $\left(a_{1}, a_{2}, a_{3}\right)$ is also unique.

Case 2. Assume that $a_{1}^{\prime}=1$ and $a_{2}^{\prime}>1$. Then $s=s_{2}=s_{3}=1$ is impossible as $a_{1}>1$. Thus the graph has $s\left(s_{2}+s_{3}\right) \geqq 3$ branches and $E(P)$ can not be blown down. By the graph $a_{2}^{1}, a_{3}^{1}, s s_{2}, s s_{3}$ are determined. Thus $s=$ g.c.d. $\left(s s_{2}, s s_{3}\right)$ is also determined. By $\left(\#_{1}\right)$ and $\left(\#_{2}\right), s_{1}=1$ and a is also recovered by these data.

Case 3. Assume that $a_{1}^{\prime}=a_{2}^{\prime}=1$ and $a_{3}^{\prime}>1$. As $a \neq\left(2,2, a_{3}\right)$ by the assumption, we must have $s=s_{1}=s_{2}=1$ by $\left(\#_{1}\right)$ and $\left(\#_{2}\right)$. Thus $a_{1}=s_{3}$ which implies $s_{3}>2$. The graph has $s_{3}$ branches at $E(P)$ and $E(P)$ can not be blown down. Thus $a_{3}$ ' and $s_{3}$ are determined from the graph, which makes to be unique.

The case that $a_{i}^{\prime}=1$ for $i=1, \ldots, 3$ are impossible from the assumption and $\left(\#_{1}\right)$ and $\left(\#_{2}\right)$. The assertion (ii) is immediate from

Lemma (6.7). Let $\Gamma$ be a star of rational curves in a complex surface $M$

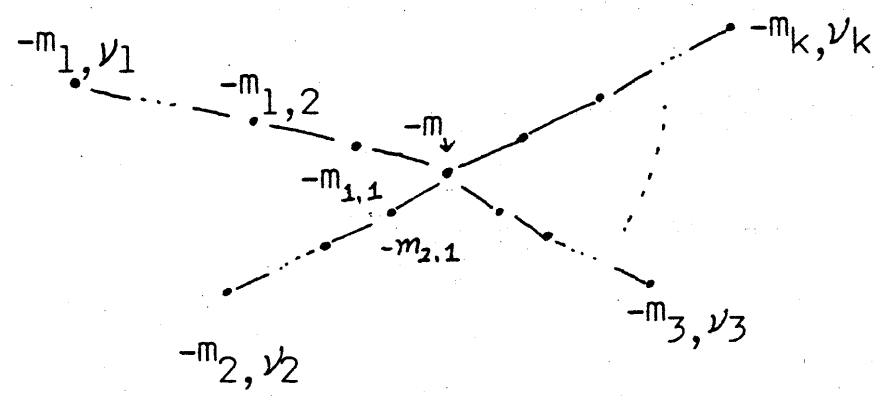

where $k \geqq 3$ and $-m_{i, j} \leqq-2$ and $-m$ 㧒e self-intersection numbers. Let $\partial N$ be the boundary of a sufficiently small tubular neighborhood of 
$\Gamma$ in $M$. Let $\frac{c_{i}}{c_{i}^{\prime}}=\left[m_{i, 1}, \ldots, m_{i, \nu}\right]$. Then $\pi_{i}(\partial N)$ is a central extension of a Dyck group $\left\langle x_{1}, \ldots, x_{k} ; x_{1}^{c_{1}}=\ldots=x_{k}^{c_{k}}=x_{1} x_{2} \cdots x_{k}=e>\right.$.

Proof. By $[13]$ or $[4], \pi_{1}(\partial N)$ can be represented by $<x, x_{i j}$ $i=1, \ldots, k, 1 \leqq j \leqq \nu_{k} ; R_{1}: x_{i, j-1} x_{i, j}^{-m_{i j}} x_{i, j+1}=e, i=1, \ldots, k$, $j=1, \ldots, \nu_{i}$ and $R_{2}: x_{1,1} x_{2,1} \ldots x_{k, 1} x^{-m}=e>$ where $x_{i, 0}=x$ and $x_{i, v_{i}+1}=e$. By $R_{1}$, we can cancel generators $x_{i j}\left(1 \leqq j \leqq \nu_{i}-1\right)$ so that

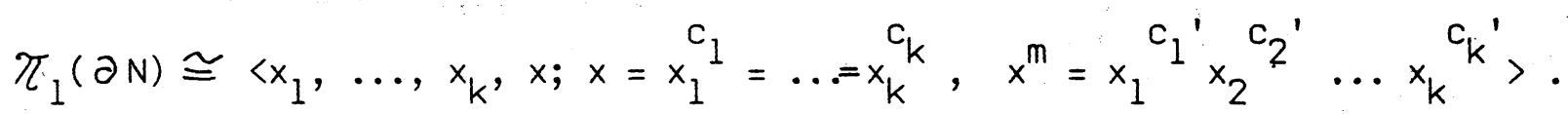
Here $x_{i}=x_{i, \nu_{i}}$. Clearly $x$ is an element of the center of $\pi_{1}(\partial N)$ and $\pi_{1}(\partial N) /\langle x\rangle$ is isomorphic to

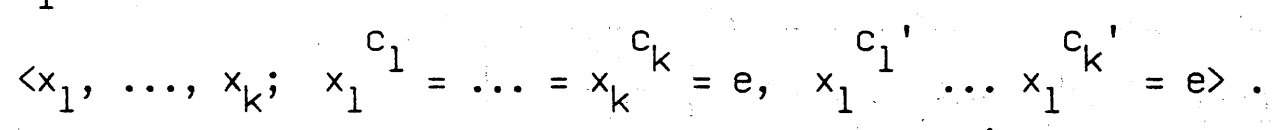

As $c_{i}$ and $c_{i}{ }^{\prime}$ are coprime, we can take $y_{i}=x_{j}{ }^{c^{\prime}}$ as generators. Thus $\pi_{1}(\partial N) /\langle x\rangle$ is isomorphic to the Dyck group

$$
\left\langle y_{1} \ldots y_{k} ; y_{1}^{c_{1}}=\ldots=y_{k}{ }^{c_{k}}=y_{1} \ldots y_{k}=e\right\rangle \text {. }
$$

For the other geometry of $k_{a}$, see $[17]$ and $[11]$.

(II). Let $f=z_{1}{ }^{a_{1}}+z_{1}\left(z_{2}{ }^{a_{2}}+z_{3}{ }^{a_{3}}\right)+b z_{2}{ }^{s} z_{3}^{t}$ where $b$ is a generic constant and $s>0, t>0$. We assume that $f$ is a weighted homogeneous by the weights $P=\left(p_{1}, p_{2}, p_{3}\right)$. Thus we have

(*) $p_{1} a_{1}=p_{1}+p_{2} a_{2}=p_{1}+p_{3} a_{3}=s^{\prime} p_{2}+t^{\prime} p_{3}$.

This class of weighted homogeneous polynomials is omitted in the classification 
table of Orlik-Wagreich [ 17 ] and this class is special in the sense that the corresponding $s^{1}$-action on $k=f^{-1}(0) \cap S^{5}$ has four singular orbit types. (For the other classes, there are three orbit types.) In fact, the dual Newton diagram is

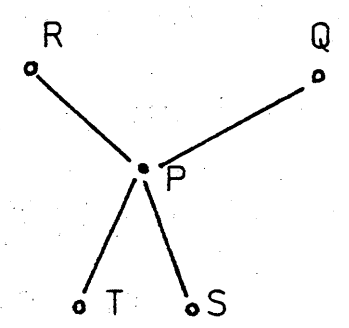

$$
\begin{aligned}
& T={ }^{t}(t, 0,1) \\
& S={ }^{t}(s, 1,0) \\
& Q={ }^{t}(0,1,0), R={ }^{t}(0,0,1)
\end{aligned}
$$

Surgery data are

\begin{tabular}{|l|c|c|c|}
\hline & $c$ & $c_{1}$ & $r+1$ \\
\hline$P Q$ & $\left(p_{1}, p_{3}\right)$ & $1+c_{1} p_{2} \equiv 0(c)$ & $\frac{a_{3} c}{p_{1}}$ \\
\hline$P R$ & $\left(p_{1}, p_{2}\right)$ & $1+c_{1} p_{3} \equiv 0(c)$ & $\frac{a_{2} c}{p_{1}}$ \\
\hline$P T$ & $p_{2}$ & $1+c_{1} p_{3} \equiv 0(c)$ & 1 \\
\hline$P S$ & $p_{3}$ & $1+c_{1} p_{2} \equiv 0(c)$ & 1 \\
\hline
\end{tabular}

$g(E(P))=\frac{a_{1} a_{2} a_{3}+s a_{3}+t a_{2}}{2 p_{1} a_{1}}-\frac{1}{2}\left\{\frac{a_{3}}{p_{1}}\left(p_{1}, p_{3}\right)+\frac{a_{2}}{p_{1}}\left(p_{1}, p_{2}\right)\right\}+1$.

Example (6.8). $f=z_{1}^{13}+z_{1}\left(z_{2}^{18}+z_{3}^{8}\right)+z_{2}^{6} z_{3}^{6}$.

$f$ is'weighted homogeneous, by the weright $P=(6,4,9)$.

The resolution graph is 


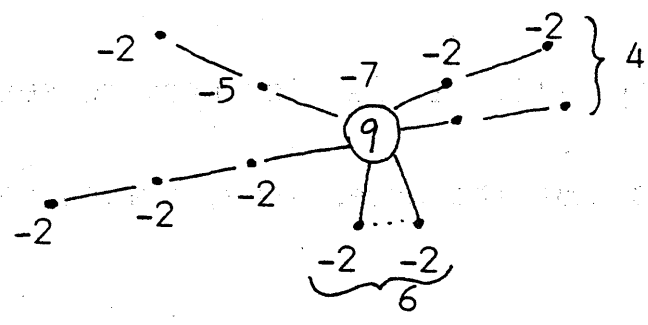

The other classes of weighted homogeneous polynomials can be treated in the same way.

(B). $T_{p, q, r}$ singularity. $\left(\left[\begin{array}{ll}1 & ]\end{array}\right)\right.$.

Let $f=z_{1}^{p}+z_{2}^{q}+z_{3}{ }^{r}+z_{1} z_{2} z_{3}$ where $\frac{1}{p}+\frac{1}{q}+\frac{1}{r}<1$. Let $P={ }^{t}\left(p_{1}, p_{2}, p_{3}\right), Q={ }^{t}\left(q_{1}, q_{2}, q_{3}\right)$ and $R={ }^{t}\left(r_{1}, r_{2}, r_{3}\right)$ be the vertices of $\Gamma^{*}(f)$ which correspond to the supports of $z_{2}{ }^{q}+z_{3}{ }^{r}+z_{1} z_{2} z_{3}$, $z_{1}^{p}+z_{3}{ }^{p}+z_{1} z_{2} z_{3}$ and $z_{1}^{p}+z_{2}^{q}+z_{1} z_{2} z_{3}$ respectively. They satisfy

(*) $\left\{\begin{array}{l}p_{2} q=p_{3} r=p_{1}+p_{2}+p_{3} \\ q_{1} p=q_{3} r=q_{1}+q_{2}+q_{3} \\ r_{1} p=r_{2} q=r_{1}+r_{2}+r_{3}\end{array}\right.$

Thus $S_{2} \Gamma^{*}(f)$ is

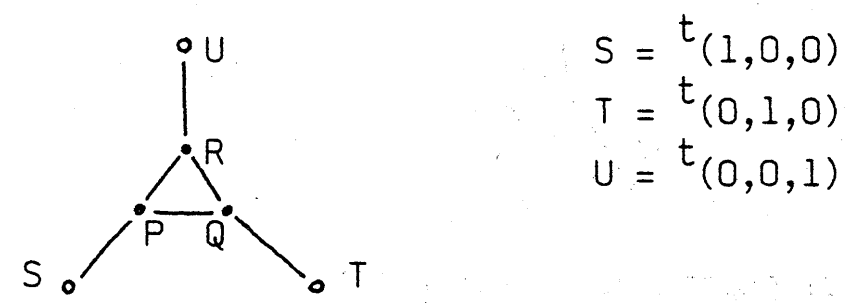

First observe that $g(\Delta(P))=g(\Delta(Q))=g(\Delta(R))=0$. Next we have 
$\operatorname{det}(P, S)=g \cdot c \cdot d\left(p_{2}, p_{3}\right)=1$ by $(*)$. It is also easy to see that $r(P, Q)=0$. Thus after blowing down the exceptional curves of the first kind, we get

Proposition (6.9). The resolution graph of $T_{p, q, r}$ is a cyclic chain of raitonal curves.

Example (6.10). $T_{5,6,7}: x^{5}+y^{6}+z^{7}+x y z=0, P=(29,7,6)$, $Q=(7,23,5), R=(6,5,19)$. The resolution graph by the theorem is

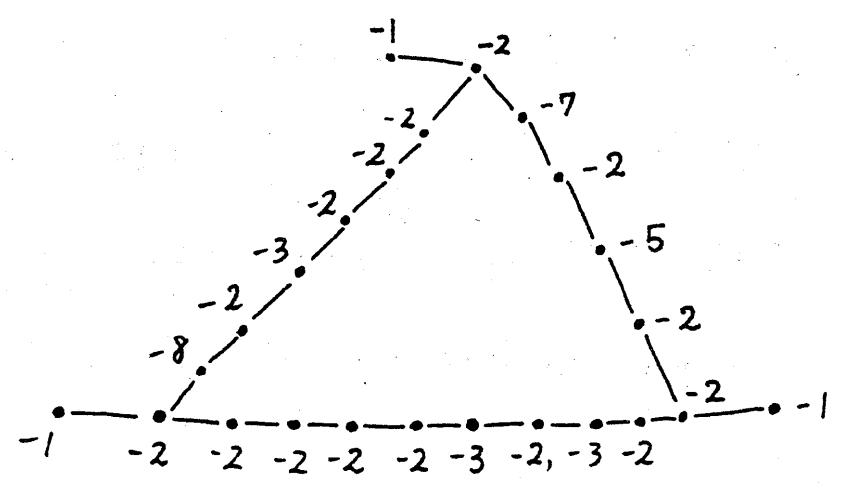

By blowing down, the graph reduces to

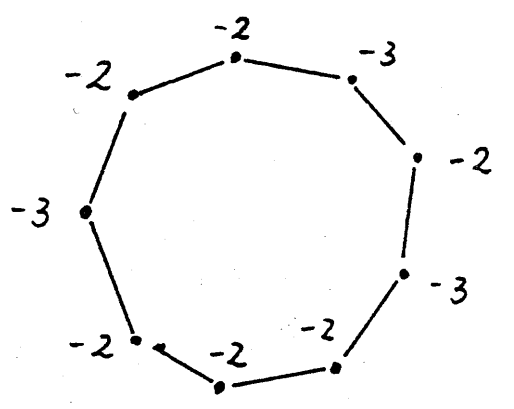

(C). Join tyree singularity. ([18], [16])

Let $g(x, y)$ be an analytic function with a non-degenerate Newton boundary. We assume that $g^{-1}(0)$ has an isolated singularity at the origin. Let $Q_{i}=\left(a_{i}, b_{i}\right) \quad(i=1, \ldots, k)$ be the vertices of $\Gamma(g)$ and let 
$\Delta_{i}=\bar{Q}_{i-1} Q_{i}(i=1, \ldots, k)$. We assume that $a_{0}<a_{1}<\cdots<a_{k}$, and $b_{0}>b_{1}>\ldots>b_{k} \cdot$ Let $r(x, y, z)=g(x, y)+z^{a}$ for an integer

a $>1$. The Newton boundary $\Gamma(f)$ has $k$ 2-simplexes $\widetilde{\Delta}_{i} \quad(i=1, \ldots, k)$ which correspond to $\left\{\Delta_{i}\right\}$ and $\Gamma_{*}^{*}(f)$ looks like this:

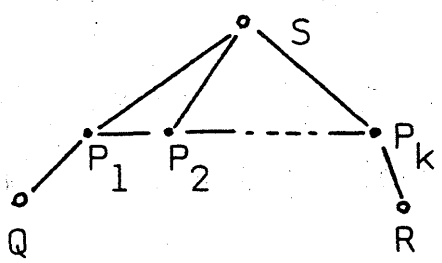

$$
S=\left(\begin{array}{l}
0 \\
0 \\
1
\end{array}\right)
$$

$\left(Q\right.$ is either $t_{(1,0,0)}$ or $t_{(a, 0,1)}$ depending whether or ${ }^{t}=a_{0}$ )

Thus the resolution graph $\Gamma$ of $v=f^{-1}(0)$ is of the following type.
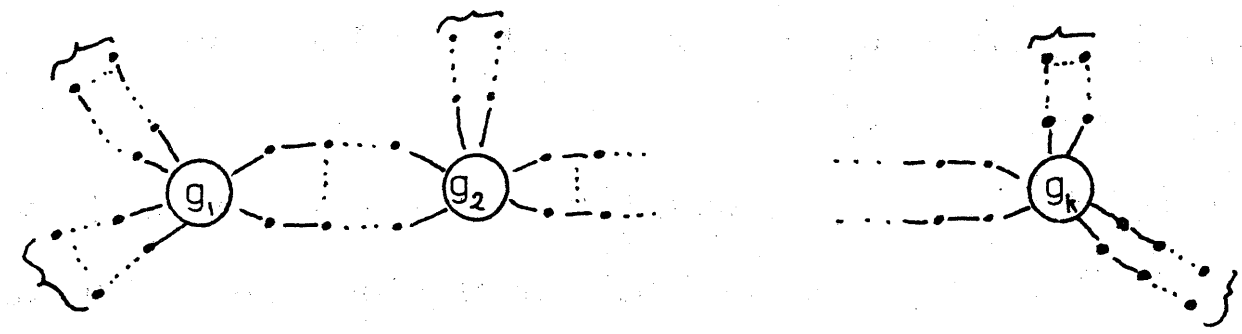

We look for the necessary and sufficient condition for $\Gamma$ to be a tree of rational curves. We must have

(i) $r\left(P_{i}, P_{i+1}\right)=0$ for $i=1, \ldots, k-1$ and

(ii) $g\left(P_{i}\right)=0$ for $i=1, \ldots, k$.

The condition ( $i$ ) is equivalent to

(i)' g.c.d. $\left.a, a_{i}, b_{i}\right)=1$ for $i=1, \ldots, k-1$.

By an easy calculation using Corollary (6.5), we have

$g\left(P_{i}\right)=\frac{1}{2}\left\{m_{i}\right.$ g.c.d.(a, $\left.\left.d_{i}\right)-g \cdot c \cdot d \cdot\left(a, a_{i-1}, b_{i-1}\right)-g \cdot c \cdot d \cdot\left(a, a_{i}, b_{i}\right)-m_{i}\right\}+1$

where $m_{i}=$ g.c.d. $\left(a_{i}-a_{i-1}, b_{i-1}-b_{i}\right)$ and $\quad d_{i}=\left(a_{i} b_{i-1}-a_{i-1} b_{i}\right) / m_{i}$. 
Note that $g_{\Delta_{i}}(x, y)$ is a weighted homogeneous polynomial of degree $d_{i}$ with weights $\left(\frac{b_{i-1}-b_{i}}{m_{i}}, \frac{a_{i}-a_{i-1}}{m_{i}}\right)$. Thus for $2 \leqq i \leqq k-1, \quad g\left(P_{i}\right)=0$ (under (i)) if and only if g.c.d(a, $\left.d_{i}\right)=1$. As $g$ has an isolated critical point at the origin, $a_{0}=0$ or 1 and $b_{k}=0$ or 1 . We assume that $\left(a_{0}, b_{k} \neq\right.$ $(0,0)$ if $k=1$ by (I). We consider $g\left(P_{1}\right)$. If $a_{0}=1$, we have g.c.d(a, $\left.a_{0}, b_{0}\right)=1$ and therefore g.c.d(a, $\left.d_{1}\right)=1$. Assume that $a_{0}=0$. As $m_{1}=\left(a_{1}, b_{0}-b_{1}\right)$ and $d_{1}=\frac{a_{1} b_{0}}{m_{1}}$, we have $g\left(P_{1}\right) \geqq \frac{1}{2}\left(m_{1}-1\right)\left\{g \cdot c \cdot d\left(a, d_{1}\right)-1\right\}$. Thus $g\left(P_{1}\right)=0$ if and only if either g.c.d $\left(a, b_{0}\right)=\operatorname{g.c} \cdot d\left(a, d_{1}\right)=1$ or $\left(a_{1}, b_{0}-b_{1}\right)=1$ and $\left(a, b_{0}\right)=\left(a, a_{1} b_{0}\right)$. Thus we obtain

Theorem (6.11). (Compare with $[9])$. Assume that $g$ is not of Brieskorn type. Then $\Gamma$ is a tree of rational curves if and only if the following are satisfied.

(i) $\operatorname{g.c} . d\left(a, a_{i}, b_{i}\right)=1$ for $i=1, \ldots, k-1$.

(ii) g.c.d $\left(a, d_{i}\right)=1$ for $i=2, \ldots, k-1$.

(iii) If g.c.d(a, $\left.a_{0}, b_{0}\right)=1$, g.c.d $\left(a, d_{1}\right)=1$.

If $g . c . d\left(a, a_{0}, b_{0}\right)>1, g . c . d\left(a, b_{0}\right)=g . c . d\left(a, a_{1} \cdot b_{0}\right)$ and $\left(a_{1}, b_{0}-b_{1}\right)=1$.

(iv) If g.c.d(a, $\left.a_{k}, b_{k}\right)=1$, g.c.d(a, $\left.d_{k}\right)=1$. 
If g.c.d(a, $\left.a_{k}, b_{k}\right)>1$, g.c.d $\left(a, a_{k}\right)=\operatorname{g.c.d}\left(a, b_{k-1} a_{k}\right)$ and

g.c.d $\left(a, a_{k}-a_{k-1}\right)=1$.

Remark (6.12). $\Gamma$ is a tree of rational curves if and only if the neighborhood boundary $K$ of the exceptional divisors if a $Q$-homology sphere ([ 4 ]). The latter condition is equivalent to: 1 is not an eigenvalue of the monodromy of $f$. Thus Theorem (6.11) can also be proved through [ 19 ].

Example (6.12). Let $g(x, y)=x^{5}+x^{2} y^{2}+y^{5}$. Then we have $m_{1}=m_{2}=1$ and $d_{1}=d_{2}=10$. $\Gamma$ is a tree of rational curves if and only. if g.c.d $(a, 10)=1$ or 5 . Here are some examples.

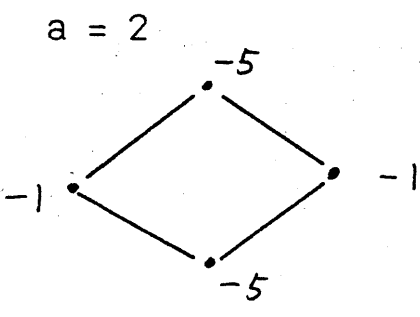

$a=5$

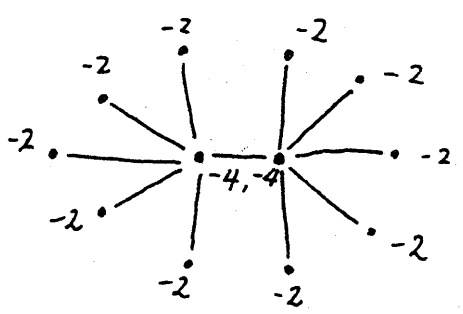

§7. Three dimensional singularity.

Let $V=f^{-1}(0)$ be as in $\$ 4$ and we assume that $n=3$. In this section, we study the topology of the exceptional divisors $E(P)$. We have three types

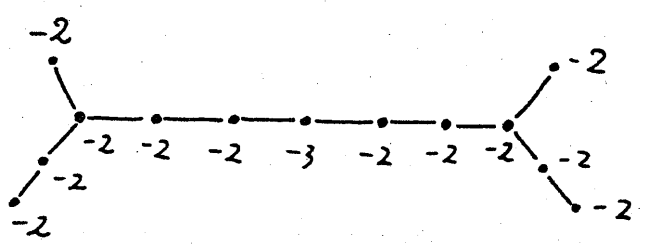

$a=10$

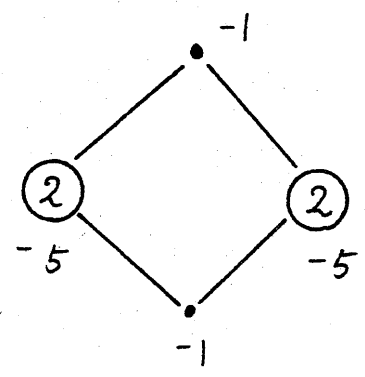


of exceptional divisors: The dimension of $\Delta(P)$ is either 1,2 , or 3 . We are particularly interested in the case that $\operatorname{dim} \Delta(P)=3$.

(I). Assume that $\operatorname{dim} \Delta(P)=1$ or $\operatorname{dim} \Delta(P) *=3$.

Let $\sigma_{1}, \ldots, \sigma_{k}$ be two simplexes in $\Delta(P)^{*}$ which contain $P$ as a vertex.

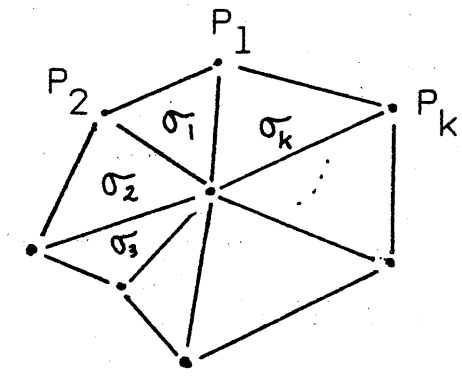

By Lemma (4.8) and Theorem (5.3), $E(P)$ has $r(\Delta(P))+1$ connected components $E(P)_{i}(i=1, \ldots, r(\Delta(P))+1)$ which are birationally equivalent to $P^{2}$ and

(7.1) $\quad \chi\left(E(P)_{i}\right)=k$.

Note that $k$ is greater than or equal to 3 .

(II). Assume that $\operatorname{dim} \Delta(P)=2(\operatorname{dim} \Delta(P) *=2)$.

Let $P^{\prime}$ and $P^{\prime \prime}$ be vertices of $\Sigma^{*}$ which are on $\Delta(P) *$ and are adjacent to $P$. Let $C(P)$, be the curve defined by $E(P) \cap E\left(P^{\prime}\right)$. By Lemma (6.4) and the calculation in the proof of Theorem (6.1), we have (7.2) $\quad g(C(P))=g(\Delta(P))$. Let $\Xi_{1}, \ldots, \Xi_{t}$ be 1-dimensional faces of $\Delta(P)$. Then $\Delta(P)^{*}$ is in the boundaries of $\Xi_{i}^{*}$ for $i=1, \ldots, t$ in $\Gamma^{*}(f)$. Let $\sigma_{i, 1}, \ldots, \sigma_{i, \nu_{i}}$ be the 2-simplexes in $\Sigma^{*}$ which are adjacent to $P$ and $\sigma_{i j} \subset$ closure $\left(\Xi_{i}^{*}\right)$

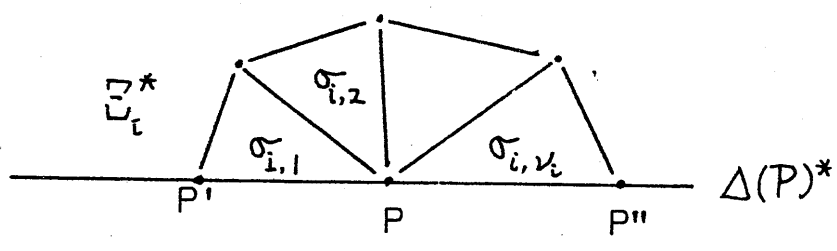


Thus by Theorem (6.1), we have

(7.3) $X(E(P))=-4 g(\Delta(P))+4+\sum_{i=1}^{t}\left(\nu_{i}-2\right)\left(r\left(\Xi_{i}\right)+1\right)$.

Note that $\nu_{i} \equiv 2$ in general so that $X(E(P)) \geqq 2(2-2 g(\Delta(P))$.

If $\nu_{i}=2$ for $i=1, \ldots, t$, the equality holds and $E(P)$ canonically projects to $C(P)$ with the fiber $P^{1}$.

(III). Assume that $\operatorname{dim} \Delta(P)=3$.

Let $\Delta_{1}, \ldots, \Delta_{s}$ be 2 -dimensional faces of $\Delta(P)$ and let

$\Xi_{1}, \ldots, \Xi_{q}$ be 1-dimensional faces of $\Delta(P)$. Let $\nu_{i}$ be the number of 2-simplexes in $\Xi_{i}^{*}$ which contain P. By Theorem (5.3) and the calculations in (I) and (II), we have

(7.4) $\quad X(E(P))=\frac{4 ! \operatorname{vol}((0, \Delta(P))}{d(P)}-2 \sum_{i=1}^{s} g\left(\Delta_{i}\right)+2 s+$ $\sum_{j=1}^{q}\left(\nu_{j}-2\right)\left(r\left(\Xi_{j}\right)+1\right)$.

Lemma (7.5). Assume that $\Delta(P)$ is a simplex. Let $\sigma=\left(P, P_{1}, P_{2}, P_{3}\right)$ be a simplex of $\Sigma^{*}$ and let $j: E(P)^{*} \rightarrow\left(\mathbb{C}_{\sigma}^{*}\right)^{3}$ be the inclusion map. Then $\left.j_{\text {\# }}: \pi_{1}\left(E(P)^{*}\right) \rightarrow \pi_{1}\left(\mathbb{C}_{\sigma}^{*}\right)^{3}\right)$ is an isómorphism.

Proof. When we move the coefficients of $f$ keeping the non-degeneracy condition, then $E(P) *$ moves diffeomorphically as there are simultaneous smooth compactifications $E(P)$. Thus we may assume that 
$g_{\Delta}\left(y_{\sigma, 1}, y_{\sigma, 2}, y_{\sigma, 3}\right)=c+\sum_{i=1}^{3} a_{i} y_{\sigma}^{\mu}$ where $c \neq 0, a_{i} \neq 0$

$(i=1,2,3)$ and $0, \mu_{1}, \mu_{2}, \mu_{3}$ are vertices of $s\left(g_{\Delta}\right)$. Let

$h\left(y_{\sigma, 1}, y_{\sigma, 2}, y_{\sigma, 3}\right)$ be the weighted homogeneous polynomial defined by

$\sum_{i=1}^{3} a_{i} y_{\sigma}^{\mu_{i}}$. By Theorem (5.3) of $[15]$, the map $b:\left(\mathbb{C}_{\sigma}^{*}\right)^{3}-h^{-1}(0) \rightarrow\left(\mathbb{C}_{\sigma}^{*}\right)^{3} \times \mathbb{C}^{*}$

defined by $b\left(y_{\sigma}\right)=\left(y_{\sigma}, h\left(y_{\sigma}\right)\right)$ gives an isomorphism of the fundamental

groups. On the other hand, $E(P)^{*}=h^{-1}(-c)$ and $\left(C^{*}\right)^{3}-h^{-1}(0)$ is a fiber

bundle over $\mathbb{C}^{*}$ with fiber $h^{-1}(-c)$. Thus the assertion is immediate from the homotopy exact sequence of the above fiber bundle.

Remark (7.6). Lemma (7.5) will presumably hold for any $\Delta(P)$ with $\operatorname{dim} \Delta(P)=3$.

Theorem (7.8). Assume that $\Delta(P)$ is a simplex. Then the fundamental group of $E(P)$ is a finite cyclic group.

Proof. Let $\sigma=\left(P, P_{1}, P_{2}, P_{3}\right)$ be a simplex of $\Sigma *$ such that $\Delta(P) \supset \Delta\left(P_{1}\right) \supset \Delta\left(P_{2}\right) \supset \Delta\left(P_{3}\right)$ and $P_{1} \in \Delta_{1}^{*}$ and $P_{2} \in \Xi_{1}^{*}$.

(We assume that $\left.\Xi_{1} \subset \Delta_{1}\right)$. Then by Lemma $(7.5), \pi_{1}(E(P ; \sigma)$ ) is a free abelian group generated by $e_{3}=(0,0,1)$. Let $\sigma^{\prime}=\left(P, P_{1}^{\prime}, P_{2}^{\prime}, P_{3}^{\prime}\right)$ be another simplex of $\Sigma^{*}$ with $\Delta(P) \supset \Delta\left(P_{1}^{\prime}\right) \supset \Delta\left(P_{2}^{\prime}\right) \supset \Delta\left(P_{3}^{\prime}\right)$ and $\operatorname{dim} \Delta\left(P_{2}^{\prime}\right)=1$. Let $\sigma^{-1} \sigma^{\prime}=\left[\begin{array}{c|ccc}1 & * & * \\ \hdashline 0 & n^{*} \\ 0 & \Lambda^{\prime} \\ 0 & \end{array}\right]$. Write $\Lambda^{\prime}=\left(\lambda_{i j}\right)$.

the

Then by $\sqrt{\text { Van Kampen Theorem, }} \pi_{1}\left(E(P ; \sigma) \cup E\left(P ; \sigma^{\prime}\right)\right)$ is a cyclic group generated 
by $e_{3}$ with relations $\lambda_{3,1} e_{3}=\lambda_{3,2} e_{3}=0$. Note that $\lambda_{3,1}=\lambda_{3,2}=0$ if and only if $P_{1}, P_{2}^{\prime}$ are on the closure $\left(\Xi_{1}^{*}\right)$. Thus the assertion is immediate from the existence of $\sigma^{\prime}$ such that $\lambda_{3,1} \neq 0$ or $\lambda_{3,2} \neq$ group

Remark (7.9). In fact, the order of the fundamental does not depend on the choice of $\Sigma^{*}$. The verification will be done in [21].

Now we study the divisor curves on $E(P)$ which are the intersection with $E\left(P^{\prime}\right)$ such that $P^{\prime}$ are adjacent to $P$. We denote by $C\left(P^{\prime}\right)$ the divisor $E\left(P, P^{\prime}\right)$. On each $\Delta_{i}^{*}$, there is a point $P_{i}$ which is adjacent to $P$. $\Delta_{i}^{*}$ and $\Delta_{j}^{*}$ are on the boundaries of $\Xi_{k}^{*}$ if $\Delta_{i} \cap \Delta_{j}=\Xi_{k}$. We assume that $\Delta_{i}^{*}$ and $\Delta_{j}^{*}$ are on the boundaries of $\Xi_{k}^{*}$. Let $P_{k, 1}, \ldots, P_{k, \nu_{k-1}}$ be vertices of $\Xi_{k}^{*}$ which are adjacent to $P$.

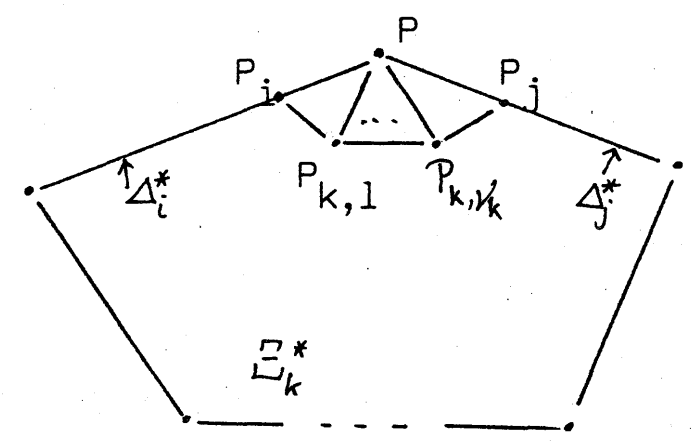

Figure (7.10)

By the calculation of the Euler characteristics, it is easy to see that each connected component of $C\left(P_{k, m}\right)$ is a rational curve and $C\left(P_{i}\right)$ has genus $g\left(\Delta_{i}\right)$. Let $P=\left(\begin{array}{c}p_{1} \\ \vdots \\ p_{4}\end{array}\right)$. To calculate the self-intersection numbers of $C\left(P_{i}\right)$ and $C\left(P_{k, m}\right)$, we consider the rational function $\varphi=\pi^{*}\left(z_{1}{ }_{2} / z_{2}^{P_{1}}\right)$ 
on $E(P)$. For any vector $Q=\left(\begin{array}{c}q_{1} \\ \vdots \\ q_{4}\end{array}\right)$, we define $|Q, P|$ by $\frac{\text { the } 2 \times 2 \text { minor }}{q_{1} p_{2}-q_{2} p_{1}}$

We assume that $\Sigma^{*}$ is canonical at $P$ in the sense of Lemma (3.9). Namely

(i) $\Sigma^{*} \mid S_{2} T^{*}(f)$ is the subdivision by the canonical primitive sequences.

(ii) $P_{k, 1}, \ldots, P_{k, \nu_{k-1}}$ are determined by $(i i-a)$ if $\operatorname{det}\left(P, P_{i}, P_{j}\right)=1$

$\nu_{k}=2$ and $P_{k, 1}=P_{i}+P_{j}$ or $(i i-b) c=\operatorname{det}\left(P_{,} P_{i}, P_{j}\right)>1$ and

(*) $P_{k, m+1}=\frac{1}{c_{m}} P_{j}+\frac{c_{m+1}}{c_{m}} P_{k, m}+\frac{d_{m+1}}{c_{m}} P \quad\left(m=0, \ldots, \nu_{k}-1\right)$

where $c_{0}=c$ and $c_{0}>c_{1}>\cdots>c_{\nu_{k-1}}=1$ and $c_{\nu_{k}}=0$ and $0 \leqq d_{m}<c_{m-1}$ $P_{k, 0}=P_{i}$ and $P_{k, \nu_{k}}=P_{j}$. Let $P_{k, m}=\left[\begin{array}{c}x_{1, m} \\ \vdots \\ x_{4, m}\end{array}\right]$.

Let $C\left(P_{k, m}\right)_{t} ; t=1, \ldots, r\left(\Xi_{k}\right)+1$ be the components of $C\left(P_{k, m}\right)$. Then by the same discussion as in $\S 6$, we have $(\varphi)=\sum_{k, m} \mid P_{k, m}, P / C\left(P_{k, m}\right)$.

Therefore we have

$$
\text { (丷) }-C\left(P_{k, m}\right)_{t}^{2}=\frac{\left|P_{k, m-1}, P\right|+\left|P_{k, m+1}, P\right|}{\left|P_{k, m}, P\right|}
$$

for $m=1, \ldots, \nu_{k}-1$. On the other hand, we can interpret (*) as:

(*)' $\quad\left|P_{k, m+1}, P\right| c_{m}=\left|P_{k, m}, P\right| c_{m+1}+\left|P_{j}, P\right|$

By the exact same discussion as in Lemma (3.6), we get 
Theorem (7.10). Assume that $\Sigma^{*}$ is canonical at $P$. Then the continuous fraction $\left[s_{1}, \ldots, s_{\nu_{k-1}}\right]$ is equal to $\frac{c}{c_{1}}$ where $s_{m}=-c\left(P_{k, m}\right)_{t}^{2}$. (If $c=1, \nu_{k}=2$ and $s_{1}=1$. Otherwise, $s_{i} \geq 2$. ) $-C\left(P_{i}\right)^{2}$ is equal to

$$
\frac{\Sigma^{\prime}\left(r\left(\Xi_{k}\right)+1\right)\left|P_{k, 1}, P\right|}{\left|P_{i}, P\right|}
$$

where $\Sigma^{\prime}$ is the sum for $k$ such that $\Xi_{k} \subset \Delta_{i}$.

Remark (7.11). If $\left|P_{i}, P\right|=0$, we take another $2 \times 2$ minor to determine the intersection numbers.

Example (7.12). Let $f\left(z_{1}, z_{2}, z_{3}, z_{4}\right)=z_{1}^{a_{1}}+z_{2}^{a_{2}}+z_{3}^{a_{3}}+z_{4}^{a_{4}}$. We assume that g.c.d. $\left(a_{i}, a_{j}\right)=1$ for any $i \neq j$. Then $P={ }^{t}\left(a_{2} a_{3} a_{4}, a_{1} a_{3} a_{4}, a_{1} a_{2} a_{4}, a_{1} a_{2} a_{3}\right)$ is the weight vector. The dual Newton diagram $\Gamma^{*}(f)$ is a 3 -simplex plus a barycenter $F$.

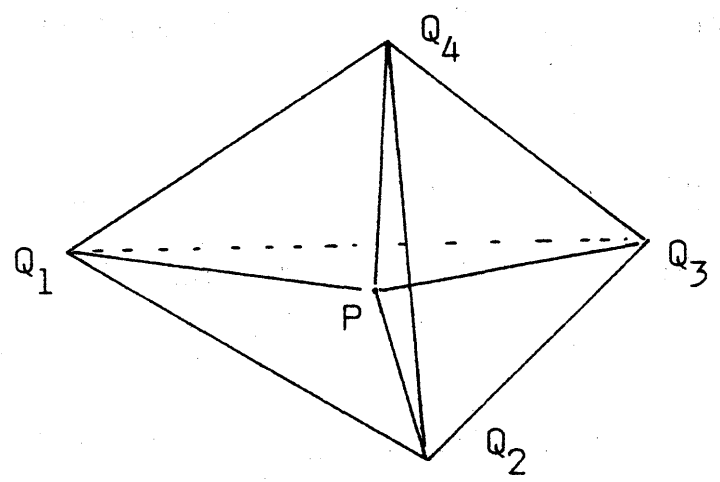

$$
\begin{aligned}
& Q_{1}={ }^{t}(1,0,0,0) \\
& Q_{2}={ }^{t}(0,1,0,0) \\
& Q_{3}={ }^{t}(0,0,1,0) \\
& Q_{4}={ }^{t}(0,0,0,1)
\end{aligned}
$$

Thus $\Gamma^{*}(f)$ has four 1 -simplexes $\overline{P Q_{i}}$ and six 2-simplexes $\Delta\left(P Q_{i} Q_{j}\right)$ at $P$. Let $\Sigma^{*}$ be a canonical simplicial subdivision. $\underbrace{\text { As }}_{\text {at } P} \operatorname{det}\left(P, Q_{i}\right)=a_{i}$, the vertex $P_{i}$ on $P Q_{i}$ which is adjacent to $P$ can be written as 
$P_{i}=\frac{1}{a_{i}} Q_{i}+\frac{a_{i}{ }^{\prime}}{a_{i}} P$ for some integer $a_{i}{ }^{\prime}, \quad 0<a_{i}{ }^{\prime}<a_{i}$. Consider the twosimplex $\Delta\left(P, Q_{i}, Q_{j}\right)(i \neq j)$. As $\operatorname{det}\left(P, P_{i}, P_{j}\right)=1$, we need only one vertex $P_{i j}=P_{i}+P_{j}$ on $\Delta\left(P, P_{i}, P_{j}\right)$ at $P$.

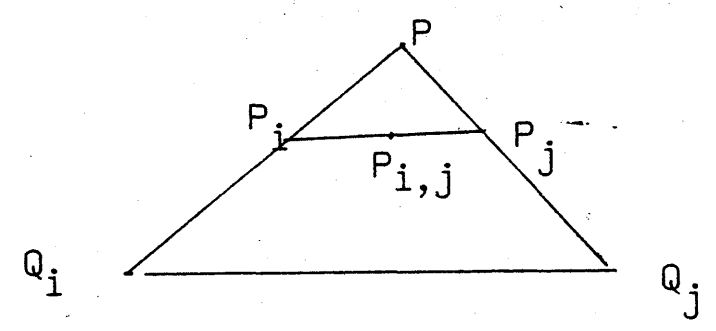

By Theorem (7.10),

$$
\begin{aligned}
x(E(P)) & =\frac{a_{1} a_{2} a_{3} a_{4}}{a_{1} a_{2} a_{3} a_{4}}+0+8+0 \\
& =9 \\
-C\left(P_{i j}\right)^{2} & =1 \\
-C\left(P_{i}\right)^{2} & =\frac{\sum_{j \neq i}\left|P_{i j}, P\right|}{\left|P_{i}, P\right|}=2
\end{aligned}
$$

We blow down 6 curves of the first kind $C\left(P_{i j}\right)(i \neq j)$ to obtain a surface $M$ of Euler characteristic $(9-6)=3$.

Assertion. $M$ is 2-dimensional projective space.

Proof. Let $\sigma=\left(P, P_{1}, P_{12}, P^{\prime}\right)$ be a simplex of $\Sigma^{*}$ such that

$$
\begin{gathered}
\Delta\left(P^{\prime}\right)=\left\{\left[\begin{array}{l}
0 \\
0 \\
0 \\
1
\end{array}\right]\right\} \text {. Then, in } \mathbf{c}_{\sigma}^{4}, E(P ; \sigma) \text { is defined by } y_{\sigma, 1}=0 \text {, and } \\
g_{\sigma}=\pi_{\sigma^{*} f / y_{\sigma, 1}} a_{1} a_{2} a^{a}{ }^{a} y_{\sigma, 2} d\left(P_{1}\right) y_{\sigma, 3} d\left(P_{i^{\prime} j}\right) y_{\sigma, 4} d\left(P^{\prime}\right)
\end{gathered}
$$

More precisely, as 3 ! volume $S\left(g_{\sigma}\right)=1$, we have 


$$
g_{\sigma}\left(y_{\sigma, 2}, y_{\sigma, 3}, y_{\sigma, 4}\right)=y_{\sigma, 2} y_{\sigma, 3}^{\alpha_{3}} y_{\sigma, 4}^{\alpha_{4}}+y_{\sigma, 3} y_{\sigma, 4}^{\beta_{4}}+y_{\sigma, 4}+1,
$$

where $\alpha_{3}, \alpha_{4}, \beta_{4}$ are positive integers.

Let $L$ be the hyperplane in $P^{3}$ defined by $x_{1}+x_{2}+x_{3}+x_{4}=0$. We define the holomorphic mapping $\psi: E(P ; \sigma) \rightarrow L$ by

$$
\text { (*) } \psi\left(y_{\sigma}\right)=\left[y_{\sigma, 2} y_{\sigma, 3}^{\alpha_{3}} y_{\sigma, 4}^{\alpha_{4}} ; y_{\sigma, 3} y_{\sigma, 4}^{\beta_{4}} ; y_{\sigma, 4} ; 1\right] . \quad \psi \text { can }
$$

also be written as $\psi\left(y_{\sigma}\right)=\left[x_{1} ; \ldots ; x_{4}\right]$ where

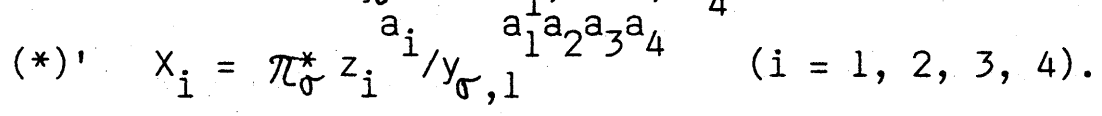

It is easy to see that $\psi$ maps $E(P ; \sigma) \cap\left(y_{\sigma, 3}=0\right)$ to a point and $\psi$ is bijective on the complement. By the second expression (*)', $\psi$ can be extended to any $E\left(P ; \sigma^{\prime}\right), \quad \sigma^{\prime}=\left(P, P_{1}^{\prime}, P_{2}^{\prime}, P_{3}^{\prime}\right)$ by

$$
x_{i}=\pi_{\sigma^{\prime}}^{*} z_{i}^{a_{i}} / y_{\sigma^{\prime}, 1}^{a_{1} a_{2} a_{3} a_{4}}
$$

As $\psi\left(C\left(P_{i j}\right)\right)=1$ point, $\quad \psi$ induces an isomorphism $\quad \psi^{\prime}: M \rightarrow L$ as is desired.

The other exceptional divisors of the resolution $\pi: \widetilde{v} \rightarrow v$ are birationally equivalent to $P^{2}$ by (I), (II) and Corollary (5.4). Thus we obtain

Theorem (7.13). Let $f(z)=z_{1}^{a_{1}}+z_{2}^{a_{2}}+z_{3}^{a_{3}}+z_{4}{ }^{a_{4}}$ and assume that $a_{i}(i=1, \ldots, 4)$ are mutually coprime. Let $\Sigma^{*}$ be a canonical simplicial subdivision of $\Gamma^{*}(f)$ and let $\pi: \widetilde{v} \rightarrow \vee$ be the associated resolution of $V$. Then the exceptional divisors are birationally equivalent to $P^{2}$. Example (7.14). Let $f(z)=z_{1}^{d}+z_{2}^{d}+z_{3}^{d}+z_{4}^{d}$. This singularity can be resolved by a single blowing up with an exceptional divisor $E_{d}$ which 
is the projective surface of degree $d$ in $P^{3}$ defined by $f=0$. This corresponds to the resolution with respect to $\Sigma^{*}=\Gamma^{*}(f)$. (The condition (2.2) is not satisfied.)

If we take a canonical simplicial subdivision $\Sigma *$ at $P(P)$-contains $6 d$ curves of the first kind and $X(E(P))=d^{3}-4(d-1)(d-2)+8$. Let $M$ be the manifold which is obtained by blowing down $6 \mathrm{~d}$ curves of the first kind. Then by the similar discussion as in Example (7.12), we have $M \cong E_{d}$. Remark (7.15). In general there is no unique minimal resolution of 3-dimensional singularity.

Example (7.16). Let $f(z)=z_{1}^{d a_{1}}+z_{2}^{d a_{2}}+z_{3}^{d a_{3}}+z_{4}^{d a_{4}}$ where $a_{i}$ are mutually coprime. Then we can take the same $\Sigma^{*}$ as in Example (7.12) as a canonical simplicial subdivision of $\Gamma^{*}(f)$. The only differences are $g\left(\Delta_{i}\right)=\frac{(d-1)(d-2)}{2}$ and $r\left(\Xi_{j}\right)+1=d$. Thus $x(E(P))=d^{3}-4(d-1)(d-2)+8$. Blow down $6 d$ curves of exceptional curves of the first kind and let $M$ be the quotient manifold. We can define an isomorphism $\psi: M \rightarrow E_{d} \subset P^{3}$ by the same equation (*)' of Example (7.12).

Further details about three dimensional singularity will be studied in $[21]$. 
References

[1] Arnold, V.I., Critical Points of Smooth Functions and their Normal Forms, Uspekhi. Mat. Nauk 30:5 (1975), 3- 65, MR \# 8701.

[2] Brieskorn, E., Beispiele zur Differentialtopologie von Singularitaten. Inventiones Math. 2 (1966) $1-14$.

[3] Durfee, A.H., The Signature of Smoothings of Complex Surface Singularities. Math. Ann. 232, $85-98$ (1978).

[4] Hirzebruch, F., The Topology of Normal Singularities of an algebraic Surface. Seminaire Bourbaki, no 250, 1962/63.

[5] Kempf, G., Knudsen, F., Mumford, D., Siant-Donat, B., Toroidal Embeddings, Lecture Notes in Math. 339, Berlin-Heidelberg-New York, Springer 1973.

[6] Kouchnirenko, A.G., Polyèdres de Newton et Nombres de Milnor. Inventiones Math. 32, 1 - 32 (1976).

[7] Laufer, H.B., On $\mu$ for surface singularities, Proceedings of Symposia in Pure Math. 45 - 49, vol. 30 (1977).

[8] Laufer, H.B., Normal Two- Dimensional Singularities. Annals of Math. Studies 71, 1971, Princeton Univ. Press.

[9] Libgober, A., Alexander Polynomial of Plane Algebraic Curves and Cyclic Multiple Planes. Duke Math. J. vol. 49, No. 4, 833 - 851 , 1982.

[10] Milnor, J., Singular Points of Complex Hypersurfaces. Annals of Math. Studies 61, 1968, Princeton Univ. Press.

[11] Milnor, J., On the three dimensiomal Brieskorn manifolds $M(p, q, r) .175-225$, in Knots, Groups, and 3-Manifolds, Annals of Math. Studies 84, 1975, Princeton Univ. Press.

[12] Milnor, J., Orlik, P., Isolated singularities defined by weighted homogeneous polynomials. Topology 9, $285-293$ (1970).

[13]Mumford, D., The topology of normal singularities of an algebraic surface and a criterion for simlicity. Publ. math. No. 9, I'Inst. des hautes 
études sci. Paris. 1961.

[14] Oka, M., On the bifurcation of the multiplicity and the topology of the Newton boundary. J. Math. Soc. Japan, vol 31 (1979), 435 - 450.

[15] Oka, M., On the topology of the Newton boundary II, J. Math. Soc. Japan, vol. $32(1980), 65-92$.

[16] Oka, M., On the homotopy types of hypersurfaces defined by weighted homogeneous polynomials. Topology vol. 12, 19 - 32 (1973).

[17] Orlik, P., wagreich, P., Isolated singularities of algebraic surface with $C^{*}$ - action. Annals of Math. 93, 205 - 228 (1971).

[18] Sebastiani, M., Thom, R., Un resultat sur la monodromie, Inventiones Math. $13(1971)$

[19] Varchenko, A.N., Zeta- Function of Monodromy and Newton's Diagram. Inventiones Math. 37, 253 - 262 (1976).

[20] Wagreich, P., Singularities of complex surfaces with solvable local fundamental group. Topology 11, 51 - 72 (1972).

[21] Oka, M., On the resolution of three dimensional hypersurface singularity, in preparation. 